\title{
BIFURCATION ANALYSIS OF AN SIR EPIDEMIC MODEL WITH SATURATED TREATMENT FUNCTION
}

\author{
by
}

Phuc Ngo, BSc, Ryerson University, 2015

A thesis presented to Ryerson University

$$
\begin{aligned}
& \text { in partial fulfilment of the } \\
& \text { requirements for the degree of } \\
& \text { Master of Science } \\
& \text { in the Program of } \\
& \text { Applied Mathematics }
\end{aligned}
$$

Toronto, Ontario, Canada, 2017

(C)Phuc Ngo, 2017 


\section{Author's Declaration}

I herby declare that I am the sole author of this thesis. This is a true copy of the thesis, including any required final revisions, as accepted by my examiners. I authorize Ryerson University to lend this thesis to other institutions or individuals for the purpose of scholarly research.

I further authorize Ryerson University to reproduce this thesis by photocopying or by other means, in total or in part, at the request of other institutions or individuals for the purpose of the scholarly research.

I understand that my thesis may be made electronically available to the public.

Phuc Ngo

Department of Mathematics

Ryerson University 


\title{
BIFURCATION ANALYSIS OF AN SIR EPIDEMIC MODEL WITH SATURATED TREATMENT FUNCTION \\ Phuc Ngo \\ Master of Science, 2017 \\ Applied Mathematics Program \\ Ryerson University
}

\begin{abstract}
In this thesis we investigate the dynamics and bifurcation of SIR epidemic models with horizontal and vertical transmissions and saturated treatment rate. It is proved that such SIR epidemic models always have positive disease free equilibria and also have three positive epidemic equilibria. The ranges of the parameters related in the model were found under which the equilibria of the models are positive. By applying the qualitative theory of planar systems, it is shown the disease free equilibria is a saddle, stable node and globally asymptotically stable. Furthermore, it is also shown that the interior equilibria are saddle, saddle node or saddle point.
\end{abstract}




\section{ACKNOWLEDGEMENTS}

I would like to start by showing my utmost appreciation towards my supervisor, Professor Kunquan Lan. It has been an absolute pleasure to have been benefited from his knowledge and encouragement. His continuous feedback since my undergraduate studies up until the completion of my master's programs has enabled me to develop the skillset I need to further pursue a $\mathrm{PhD}$ in mathematics. His dedication to teaching and creating a valuable learning environment for me and my fellow students has been extremely helpful. I thank Professor Kunquan Lan for his detail feedback on the work that I have done which helped create the final version of this thesis. Thank you for providing the tools I need to reach my goals.

I would also like to thank Dr. Ha Minh Dzung and Dr. Xi Huo for providing feedback on my work. Their time and effort allowed me to think outside the box and solve challenging problems. Furthermore, I would like to thank my

colleague Marvin Hoti for helping me with some concepts and feedback of my work.

I want to thank the professors at Ryerson who early in my graduate studies taught me several courses of mathematics including technique, graph theory and principle for applied mathematics. I would also like to thank Kathy Peter and Teresa Lee for always assisting me with department-related procedures.

Finally, I would like to thank the members of the examining committee Dr. Kunquan Lan, Dr. Silvana Ilie, Dr. Dzung Minh Ha and the Chair Professor 
Dr. Pawel Pralat for the opportunity to present my thesis. 


\section{DEDICATION}

I would like to dedicate my work to my beloved twin daughters, Jennifer and Catherine Ngo Tran, my son, Vietca Ngo and my supporting wife, Hang Tran along with my family. 


\section{Table of Contents}

Declaration $\quad$ ii

Abstract

Acknowledgements iv

Dedication vi v vi van

Preface $\quad$ x

1 Introduction 1

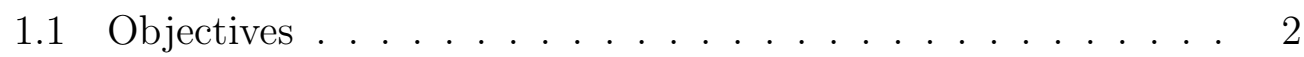

1.2 Methodology ..................... 2

1.3 Thesis organization ................ 3

2 Theory of dynamical systems 4

2.1 Introduction . . . . . . . . . . . . . . . 4

2.1.1 Definition of autonomous differential equation . . . . 5

2.1 .2 Equilibrium ................ . . 5 
2.1.3 Phase plane analysis . . . . . . . . . . . 6

2.1.4 Classification scheme of stability . . . . . . . . . 9

3 Introduction to epidemic model $\quad 12$

3.1 Introduction . . . . . . . . . . . . . . . . 12

3.2 The beginning of mathematical epidemiology . . . . . . . . . 13

3.2.1 The SIR model without vital dynamics . . . . . . . . 14

3.2.2 The SIR model with vital dynamics . . . . . . . . 15

3.3 Construction the model and modification . . . . . . . . . . 16

3.4 Previous results . . . . . . . . . . . . . . . . 17

3.5 Positive equilibria of the model (3.3.3) . . . . . . . . . . 17

4 Qualitative behaviour analysis of the equilibria points 32

$\begin{array}{lll}5 & \text { Conclusion } & 47\end{array}$

$\begin{array}{ll}\text { Bibliography } & 48\end{array}$ 


\section{List of Abbreviations}

$S$

$I$

$R$

$b$

$d$

$r$

$p$

$q$

KMK

$\frac{a y}{1+c y}$
Susceptible; individuals not infected but who are capable of contracting the disease and becoming infective.

Infected; individuals who are infected and infectious, capable of transmitting the disease to others.

Removed; individuals who have had the disease and have recovered, and who are permanently immune, or are isolated until recovery and permanent immunity occur.

The birth rate of the susceptible population, which is assumed to equal the death rate.

The natural death rate

The recovery rate of the infective individuals.

The proportion of offspring's of infective parents that are susceptible individuals and $p \in[0,1]$.

The fraction of the infected unborn or newly born offspring of the infective parent and $q \in[0,1]$.

The Kermack Mckendrick model.

The removal rate of the infected individuals. 


\section{Preface}

This thesis was writen as part of my Master of Science degree at Ryerson University. Several professors have contributed academically, practically and with support to this thesis.

Chapters 1 and 2 contain the preliminary materials, while chapters 3,4 and 5 contain the main results of the thesis. 


\section{Chapter 1}

\section{Introduction}

Many infectious diseases exist in our society such as: Chickenpox, smallpox, AIDS, H1N1... that may have tremendous effects on individuals. For example, during the Bubonic Plague also known as the Black Death, an epidemic in Europe first occurring between 1348 to 1350 about thirty to sixty percent of the European population were wiped out. In 1520 a smallpox epidemic caused half of the population of the Aztec to perish. A recent epidemic caused by severe acute respiratory syndrome (SARS), in 2003 affected more than five thousand people. Another example is the H1N1 Influenza pandemic that occurred in 2009.

Many theories were developed during the nineteenth century, from Hamer model(1906) and Ross model (1911), Anderson-May, Dietz, where these models become more concrete as time progresses. Many questions arise when our society is faced with a new type of disease such as: factors effecting the spread of the disease, which individuals to treat, how to stabilize the spread 
of the disease and how to terminate it. In order to understand these epidemic system characteristics mathematical modeling can be used.

\section{$1.1 \quad$ Objectives}

The primary objective of this thesis are:

1. Modify the previous SIR epidemic model and study the dynamical behaviours.

2. To find the conditions of the parameters involved in the model to providing the positive equilibria and study the qualitative behaviour at each equilibrium.

\subsection{Methodology}

We are interested in the study of the epidemic model with a saturated treatment function. After we modify the previous SIR epidemic model by adding the saturated treatment function $\frac{a y}{1+c y}$, we will then reduce the new system from three non-linear differential equations to two non-linear differential equations. We will find all the ranges of parameters to ensure that all equilibria are positive and then we will determine the stability of the SIR epidemic model for each of its equilibria. 


\subsection{Thesis organization}

The purpose of the thesis is to find the total number of equilibria, to investigate the phase portraits near the equilibria and to understand whether the disease will spread or persist for our model of interest.

In Chapter 1, there will a brief introduction to the epidemiology, problems and the explanations of the thesis objectives.

In Chapter 2, we will introduce the definitions, mathematical theory, terminology and methods that we will use to analyze the results of the model.

Chapter 3 and 4 contain the main results of the thesis and chapter 5 contains the conclusion of our model. 


\section{Chapter 2}

\section{Theory of dynamical systems}

\section{$2.1 \quad$ Introduction}

We will present the mathematical theories and techniques that are useful to study non-linear differential equations. It will be useful to interpret the biological meaning of the epidemic model. In order to understand and to analyze the model we need to study the equilibrium points of the system. For example, if there exists an equilibrium point of the form $(S, 0)$, then it can represent the disease-free states of an epidemic model.

We consider the following two dimensional planar systems

$$
\left\{\begin{array}{l}
\dot{x}(t)=f(x(t), y(t)), \\
\dot{y}(t)=g(x(t), y(t)),
\end{array}\right.
$$

subject to the initial value condition:

$$
(x(0), y(0))=\left(x_{0}, y_{0}\right),
$$


where $f, g \in C^{1}\left(\mathbb{R}^{2}\right)$.

\subsubsection{Definition of autonomous differential equation}

An autonomous system or autonomous differential equation is a system of ordinary differential equations has the form $\dot{x}(t)=f(x(t))$ which does not explicitly depend on the independent variable where $\mathrm{x}$ takes values in $\mathrm{n}$ dimensional and $\mathrm{t}$ is usually time.

\subsubsection{Equilibrium}

An equilibrium point of a dynamical system generated by an autonomous system of differential equations is a solution that does not change with time. In SIR model, all the parameters and equilibrium points must be positive in order to have biological meaning. If it is negative, then the solution tends to extinction.

The autonomous dynamical system has the form

$$
\left\{\begin{array}{l}
\dot{x}=f(x, y), \\
\dot{y}=g(x, y),
\end{array}\right.
$$

Definition 2.1.1. [2] A point $(\bar{x}, \bar{y}) \in \mathbb{R}_{+}^{2}$ is an equilibrium point of (2.1.3) if it satisfies $f(\bar{x}, \bar{y})=0$ and $g(\bar{x}, \bar{y})=0$. An equilibrium point $(\bar{x}, \bar{y})$ of (2.1.3) is said to be positive if $(\bar{x}, \bar{y}) \in P$ where

$$
P=\left\{(\bar{x}, \bar{y}) \in \mathbb{R}^{2}: \bar{x} \geq 0 \text { and } \bar{y} \geq 0\right\}
$$

and to be an interior equilibrium point if $\bar{x}>0$ and $\bar{y}>0$. 


\subsubsection{Phase plane analysis}

As mentioned earlier in the introduction part, we need to understand the theory behind the dynamic behaviours of a system of two differential equations. We need to study the local stability through phase plane analysis near equilibrium point. One way to do that is to linearize a non-linear system about an equilibrium point.

Definition 2.1.2. $[3,6]$ Linearization refers to finding the linear approximation to a function at a given point. In the study of dynamical systems, linearization is a method for assessing the local stability of an equilibrium point of a system of nonlinear differential equations.

It is a technique that has been used for studying linear system to analyze the behaviour of a non-linear function near the fixed point.

Let the system of (2.1.3) be a non-linear system . We will expand the function $f$ and $g$ by using Taylor formula. Now, we are going to apply a small perturbation with $u=x-\bar{x}$ and $v=y-\bar{y}$. Then

$$
\begin{aligned}
& \frac{d u}{d t}=f(\bar{x}, \bar{y})+f_{x}(\bar{x}, \bar{y}) u+f_{y}(\bar{x}, \bar{y}) v+f_{x x}(\bar{x}, \bar{y}) \frac{u^{2}}{2}+\ldots \\
& \frac{d v}{d t}=g(\bar{x}, \bar{y})+g_{x}(\bar{x}, \bar{y}) u+g_{y}(\bar{x}, \bar{y}) v+g_{x x}(\bar{x}, \bar{y}) \frac{u^{2}}{2}+\ldots
\end{aligned}
$$

where

$f_{x}(\bar{x}, \bar{y})=\left.\frac{\partial f(x, y)}{\partial x}\right|_{x=\bar{x}, y=\bar{y}}$ and $g_{x}(\bar{x}, \bar{y})=\left.\frac{\partial g(x, y)}{\partial x}\right|_{x=\bar{x}, y=\bar{y}}$ and so on.

We will neglect the partial derivatives of the terms of order greater or equal to two. Recall from definition (2.1.1), the equilibria of the system (2.1.3) are solution $(\bar{x}, \bar{y})$ that satisfy $f(\bar{x}, \bar{y})=0$ and $g(\bar{x}, \bar{y})=0$. 
The system linearized about the equilibrium $(\bar{x}, \bar{y})$ is $[6]$

$$
\frac{d A}{d t}=J A
$$

where $A=(u, v)^{T}$ and $J$ is the Jacobian matrix evaluated at the equilibrium.

Lets recall some results on phase portraits of planar systems near equilibria in the qualitative theory $[2,4,5]$. Let us denote by $A(x, y)$ the Jacobian matrix of $f$ and $g$ at $(x, y)$, that is

$$
A(x, y)=\left(\begin{array}{ll}
\frac{\partial f}{\partial x} & \frac{\partial f}{\partial y} \\
\frac{\partial g}{\partial x} & \frac{\partial g}{\partial y}
\end{array}\right)
$$

and by $|A(x, y)|$ and $\operatorname{tr}(A(x, y))$ the determinant and the trace of $A(x, y)$ respectively.

The characteristic polynomial of $A(x, y)$ is

$$
\lambda^{2}-\operatorname{tr}(A(x, y))+\operatorname{det}(A(x, y))
$$

The solutions of a planar system near its equilibria $(x, y)$ can be studied by the eigenvalues of $A(x, y)$ which can be determined by $|A(x, y)|$ and $\operatorname{tr}(A(x, y))$.

[24] The Figure 2.1 illustrates the classification schemes of a non-linear system that has been linearized to linear system . The stability diagram in the $(T, \Delta)$ plane, where $T=\operatorname{tr}(A(x, y))$ and $\Delta=\operatorname{det}(A(x, y))$, and $\Delta=T$. 


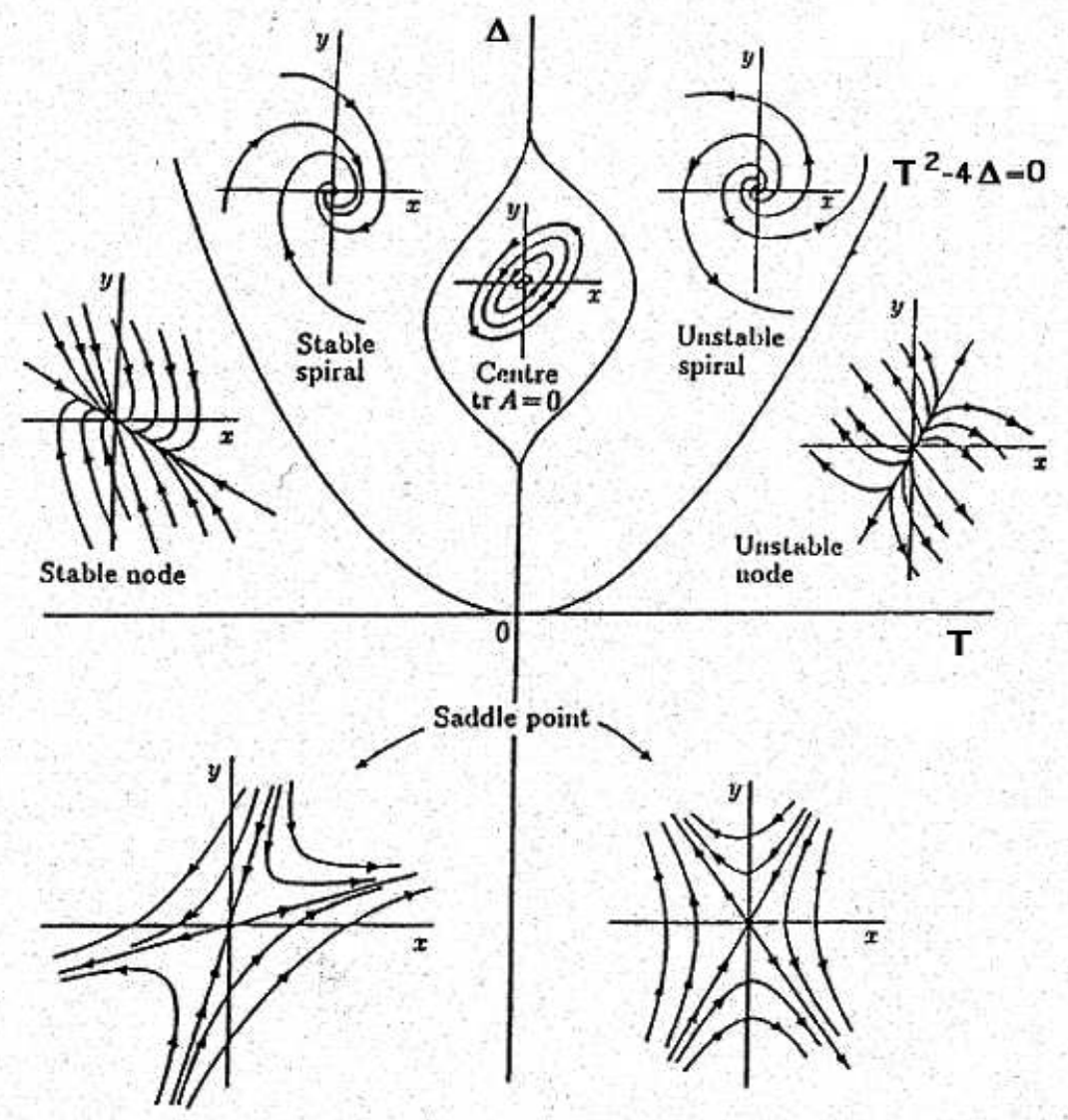

Figure 2.1: [7] The stability diagram of non-linear system. 


\subsubsection{Classification scheme of stability}

The following classification schemes can be found in $[2,4,5]$ and have been used in $[2,4,5]$.

Lemma 2.1.1. [2] If $\left(x_{0}, y_{0}\right)$ is an equilibrium of (2.1.1), then the following assertions hold.

(i) If $\left|A\left(x_{0}, y_{0}\right)\right|<0$, then $\left(x_{0}, y_{0}\right)$ is a saddle of (2.1.1).

(ii) If $\left|A\left(x_{0}, y_{0}\right)\right|>0,\left(\operatorname{tr}\left(A\left(x_{0}, y_{0}\right)\right)\right)^{2}-4\left|A\left(x_{0}, y_{0}\right)\right| \geq 0$ and $\operatorname{tr}\left(A\left(x_{0}, y_{0}\right)\right) \neq 0$, then $\left(x_{0}, y_{0}\right)$ is a node of $(2.1 .1)$; it is stable if $\operatorname{tr}\left(A\left(x_{0}, y_{0}\right)\right)<0$ and unstable if $\operatorname{tr}\left(A\left(x_{0}, y_{0}\right)\right)>0$.

(iii) If $\left|A\left(x_{0}, y_{0}\right)\right|>0,\left(\operatorname{tr}\left(A\left(x_{0}, y_{0}\right)\right)\right)^{2}-4\left|A\left(x_{0}, y_{0}\right)\right|<0$ and $\operatorname{tr}\left(A\left(x_{0}, y_{0}\right)\right) \neq$ 0 , then $\left(x_{0}, y_{0}\right)$ is a focus of $(2.1 .1)$; it is stable if $\operatorname{tr}\left(A\left(x_{0}, y_{0}\right)\right)<0$ and unstable if $\operatorname{tr}\left(A\left(x_{0}, y_{0}\right)\right)>0$.

Lemma 2.1.2. [2] Let $\left(x_{0}, y_{0}\right)$ be an equilibrium of (2.1.1). Assume that $\left|A\left(x_{0}, y_{0}\right)\right|=0, \operatorname{tr}\left(A\left(x_{0}, y_{0}\right)\right) \neq 0$ and (2.1.1) is equivalent to the following system

$$
\left\{\begin{array}{l}
\dot{u}=p(u, v), \\
\dot{v}=\varrho v+q(u, v)
\end{array}\right.
$$

with an isolated equilibrium point $(0,0)$, where $\varrho \neq 0$,

$p(u, v)=\sum_{i+j=2, i, j \geq 0}^{\infty} a_{i j} u^{i} v^{j}$ and $q(u, v)=\sum_{i+j=2, i, j \geq 0}^{\infty} b_{i j} u^{i} v^{j}$ are convergent power series. If $a_{20} \neq 0$, then $\left(x_{0}, y_{0}\right)$ is a saddle-node of (2.1.1).

The graphs and illustration of the Lemma (2.1.1) and (2.1.2) are provided below: 

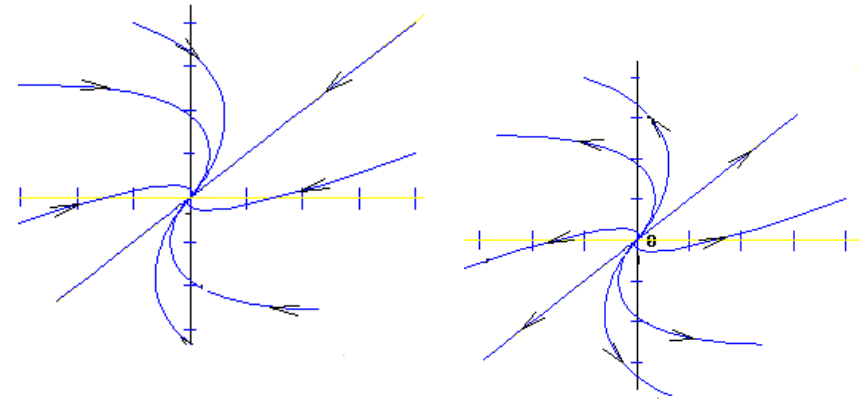

(a) Stable node - the solution will flow into the origin or the solution will approach the equilibrium regardless of the starting point.

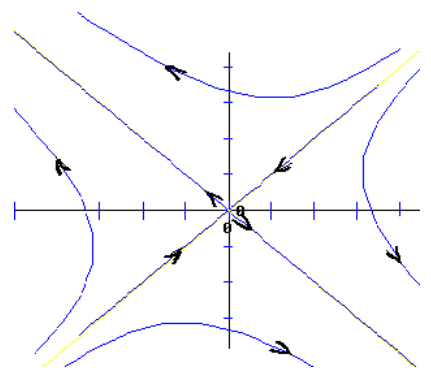

(d) Saddle point -the solution will not converge to the equilibrium (b) Unstable node -the solution will not converge to the equilibrium, unless it starts at the equilibrium.

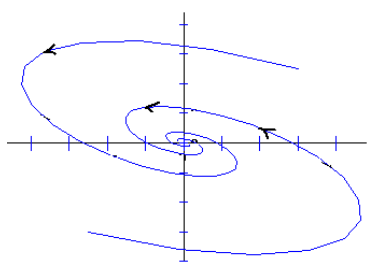

(e) Stable Spiral -the solution starts from any point other than the equilibrium, it spirals into the equilibrium . librium.

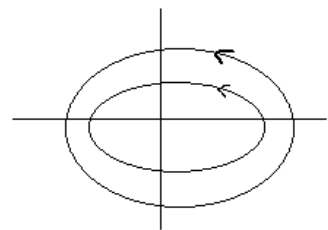

(c) Center -the solution will neither approach nor move away from the equilibrium

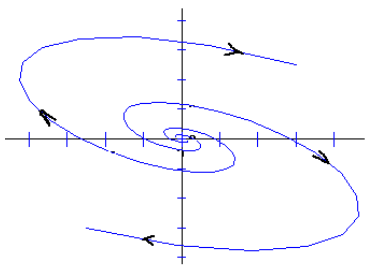

(f) Unstable Spiral -the solution starts from the fixed point and then spirals away from the equi-

Figure 2.2: Phase portrait diagram 
Lemma 2.1.3. [23] Assume that each positive solution of (2.1.1)-(2.1.2) with $\left(x_{0}, y_{0}\right) \in \mathbb{R}_{+}^{2}$ is contained in a bounded closed subset $B$ of $\mathbb{R}^{2}$. Assume that $B$ contains only one equilibrium $\left(x^{*}, y^{*}\right)$ of the system (2.1.1) and $\left(x^{*}, y^{*}\right)$ belongs to the boundary of $B$. Then each positive solution of (2.1.1)-(2.1.2) converges to $\left(x^{*}, y^{*}\right)$. 


\section{Chapter 3}

\section{Introduction to epidemic model}

\subsection{Introduction}

The first section of this chapter is devoted to the back ground of the epidemic

models. In disease modelling, there are two types of models: Simple and detail. In the simple epidemic model a constant population size $\mathrm{N}$ is assumed and there are no deaths or movement of population to affect the population size. Also, many epidemics have short time life span as compared to the life span of the individuals in the population so the population size $\mathrm{N}$ is constant.

Detail models are designed for specific circumstances including short term prediction and allow for a variety of situations such as vaccinations and quarantine.

The first introduces the SIR model which is simple and ignores vital dynamics and SIR model with vital dynamics. This will include the mechanism 
of the model and its first pioneer that came up with the model. In the second part of this chapter we will present our model of interest. The model is an expansion of the work from [8]

\subsection{The beginning of mathematical epidemi- ology}

1. Daniel Bernoulli formulated and solved a model for smallpox in 1760 . Using his model, he evaluated the effectiveness of inoculating of healthy people against the smallpox virus.

2. William Hamer formulated and analyzed a discrete time model in 1960 to understand the recurrence of measles epidemics.

3. Ronald Ross developed differential equation models for malaria as a host vector disease in 1911.

4. Kermack and McKendrick introduced a new epidemic model known as an SIR model, which is also commonly referred to as the Kermack McKendrick model (KMK). 


\subsubsection{The SIR model without vital dynamics}

The following is the model of Kermack and McKendrick ( The SIR model without vital dynamics)

$$
\left\{\begin{array}{l}
\frac{d S}{d t}=-\beta \frac{S I}{N}, \\
\frac{d I}{d t}=\beta \frac{S I}{N}-r I, \\
\frac{d R}{d t}=r I,
\end{array}\right.
$$

The next assumption of this simple model is that the population is subdivided into three subgroups $S, I$ and $R$ where:

$S \quad$ Susceptible; individuals not infected but who are capable of contracting the disease and becoming infective.

I Infected; individuals who are infected and infectious, capable of transmitting the disease to others.

$R \quad$ Removed; individuals who have had the disease and have recovered, and who are permanently immune, or are isolated until recovery and permanent immunity occur.

$N \quad$ Total population size.

$\frac{\beta}{N} S \quad$ The average number of adequate contacts made by an infected individual resulting in an infection of a susceptible individual per time.

$\frac{\beta}{N} S I \quad$ The number of infections caused by all infected individuals per time.

$\beta \quad$ The average number of adequate contacts made by an infected individual per time. 
$r$

$\mu$
The recovery rate of the infective individuals.

The per capita death rate, and the population level birth rate.

\subsubsection{The SIR model with vital dynamics}

The differential equations for the SIR model with vital dynamics is

$$
\left\{\begin{array}{l}
\frac{d S}{d t}=\mu-\beta S I-\mu S \\
\frac{d I}{d t}=\beta S I-r I-\mu I, \\
\frac{d R}{d t}=r I-\mu R
\end{array}\right.
$$

There are some drawbacks in the two classic models (3.2.1) and (3.2.2):

1. The total population size remains constant in the two classic models

2. They assume that the population is uniform and homogeneously mixing. However, mixing depends on many factors including age which is not taken into account by any of the model.

3. The model (3.2.1) assumes no birth-rate or natural death-rate, or treatment. The model SIR without vital dynamics assumes only horizontal transmission. That is individuals can only be infected by direct contact.

A new SIR epidemic model with vertical and horizontal transmission is investigated by Xinzhu Meng and Lansun Chen [10]. The vertical transmission is the passing of the infection from from a parent to an unborn offspring.

In [10], they also introduced a birth rate $b$ which is also equal to the death 
rate $d$

The following sections introduces the model of Xinzhu Meng and Lansun Chen [10] and how it is constructed, and our modification on the model.

\subsection{Construction the model and modification}

Following is the model (2.1) in [10]

$$
\left\{\begin{array}{l}
\dot{S}=-\beta S I-d S+p b I+b(S+R), \\
\dot{I}=\beta S I-d I-r I+q b I \\
\dot{R}=r I-b R
\end{array}\right.
$$

The terminology of all parameters were defined earlier and the population has a constant size. We can normalize the population, so $S(t)+I(t)+R(t)=1$. $\mathrm{S}, \mathrm{I}, \mathrm{R}$ are taken as proportions and using $S+I=1-R$. This together with $p+q=1$ reduce the system (3.3.1) to following 2-dimensional system

$$
\left\{\begin{array}{l}
\dot{x}=b-\beta x y-b x-q b y:=f(x, y), \\
\dot{y}=\beta x y-b y-r y+q b y:=g(x, y),
\end{array}\right.
$$

where $\mathrm{x}(\mathrm{t})=\mathrm{S}(\mathrm{t})$ and $\mathrm{y}(\mathrm{t})=\mathrm{I}(\mathrm{t})$.

We modify the model by adding a saturated treatment function $\frac{a y}{1+c y}$ to the second equation of the system (3.3.2). Then system (3.3.2) becomes:

$$
\left\{\begin{array}{l}
\dot{x}=b-\beta x y-b x-q b y:=f(x, y) \\
\dot{y}=\beta x y-b y-r y+q b y-\frac{a y}{1+c y}:=g(x, y)
\end{array}\right.
$$

where $a \geq 0, c \geq 0$. 


\subsection{Previous results}

The SIR models with $a=q=0$ then model (3.3.3) is the SIR model with horizontal transmissions and it was considered by Hethcote [9].

When $a=0$ and $\mathrm{q} \in(0,1)$ then model (3.3.3) is the SIR model only with horizontal and vertical transmissions and it was considered by Meng and Chen [10]. As well as Luo, Zhu and Lan with constant treatment rates [11]. In [10], they showed that the dynamical system (3.3.2) has two equilibrium points. The first one is disease free equilibrium $\left(S_{0}^{*}, I_{0}^{*}\right)=(1,0)$ and the

second equilibrium point is $\left(S_{1}^{*}, I_{1}^{*}\right)=\left(\frac{1}{R_{0}}, \frac{b\left(R_{0}-1\right)}{\beta+(b-p d) R_{0}}\right)$ where $R_{0}=\frac{1}{p d+r}=\frac{1}{S_{1}^{*}}$ is a basic reproductive number of the epidemic.

They proved that the disease-free equilibrium $\left(S_{0}^{*}, I_{0}^{*}\right)$ is unstable or locally stable under some conditions of the parameters. They also proved that the positive interior equilibrium $\left(S_{1}^{*}, I_{1}^{*}\right)$ can be unstable or locally stable.

\subsection{Positive equilibria of the model (3.3.3)}

In this section, we will find all the equilibria of (3.3.3) and provide conditions on the parameters involved in (3.3.3) under which the equilibria are positive. Recall that $(x, y) \in \mathbb{R}^{2}$ is an equilibrium of (3.3.3) if it satisfies $f(x, y)=0$ and $g(x, y)=0$. An equilibrium point $(x, y)$ is said to be positive if $x, y \geq 0$; it is a positive interior equilibrium if $x, y>0$. 
Consider the following system

$$
\left\{\begin{array}{l}
b-\beta x y-b x-b q y=0 \\
\beta x y-b y-r y+b q y-\frac{a y}{1+c y}=0
\end{array}\right.
$$

Notation: Let

$$
\eta:=\eta(b, r, q)=b(1-q)+r, \quad q_{1}=(b+r-\beta) b^{-1} .
$$

We need the following necessary and sufficient conditions of the parameters before find all the positive equilibria.

Lemma 3.5.1. (1) $\beta<\eta$ if and only if either $r \leq \beta<b+r$ and $0 \leq q<q_{1}$ or $\beta<r$ and $0 \leq q \leq 1$.

(2) $\eta<\beta$ if and only if $b+r<\beta$ and $0 \leq q \leq 1$ or $r<\beta \leq b+r$ and $q_{1}<q \leq 1$.

(3) $\eta \leq \beta$ if and only if either $b+r<\beta$ and $0 \leq q \leq 1$ or $r \leq \beta \leq b+r$ and $q_{1} \leq q \leq 1$

(4) $\eta \leq \beta<\eta+b$ if and only if either $b+r<\beta<2 b+r-q b$ and $0 \leq q \leq 1$ or $r \leq \beta \leq b+r$ and $q_{1} \leq q \leq 1$.

(5) $\beta=\eta$ if and only if $r \leq \beta \leq b+r$ and $q=q_{1}$.

Notation: Let

$$
\eta=b+r-b q, q_{1}=\frac{b+r-\beta}{b} .
$$

Proof. From the definition of $\eta$ then

$$
\begin{aligned}
\eta-\beta & =b+r-b q-\beta=(b+r-\beta)-b q \\
& =b\left[\frac{b+r-\beta}{b}-q\right]=b\left(q_{1}-q\right)
\end{aligned}
$$


and

$$
\eta-\beta+b=b\left(q_{1}-q\right)+b=b\left(q_{1}-q+1\right)
$$

1) It is easy to verify if $r \leq \beta<b+r$ then $0<q_{1}<1$ and if $\beta=r$ then $q_{1}=1$. This together with $0 \leq q<q_{1}$ and equation (3.5.2) imply the result. If $\beta<r$ then $q_{1}>1>q$ since $q \in[0,1]$. This together with (3.5.2) imply the result.

2) If $b+r<\beta$ then

$$
q_{1}=\frac{b+r-\beta}{b}<0
$$

imply

$$
q_{1}-q<0-q<0
$$

since $q \in[0,1]$. This together with equation (3.5.2) imply the result.

If $r<\beta \leq b+r$ then $0 \leq q_{1}<1$. This together with $q_{1}<q \leq 1$ and equation (3.5.2) imply the result.

3) If $b+r<\beta$ then

$$
b+r-b q<\beta-b q<\beta
$$

or $\eta<\beta$. This together with $q=q_{1}$ and equation (3.5.2) imply the result.

4) If $b+r<\beta$ we obtained $\eta<\beta$ and $q_{1}<0$ from (3). Moreover

$$
2 b+r-b q=b+r-b q+b=\eta+b
$$

so if $\beta<2 b+r-b q$ then $\beta<\eta+b$ then the result holds.

If $r \leq \beta \leq b+r$ then $0 \leq q_{1} \leq 1$ and $1-q \geq 0$ since $q \in[0,1]$. The result follows from equation (3.5.3).

5) If $r \leq \beta \leq b+r$ then $0 \leq q_{1} \leq 1$. This together with $q=q_{1}$ and equation (3.5.2) imply $\eta=\beta$ 
Lemma 3.5.2. Assume that $a, b, c, r, \beta>0$ and $q \in[0,1]$ then

1) Disease equilibrium $(1,0)$ always exists.

2) If $(x, y) \in \mathbb{R}_{+}^{2}$ is an positive equilibrium of (3.3.3), then $y$ satisfies the following equation

$$
\beta(b+r) c y^{2}+[\beta(a+b+r)-b(\beta-\eta) c] y+b(a-\beta+\eta)=0 .
$$

3) Equation (3.5.4) is equivalent to the following equation

$$
\left(y+\frac{[\beta(a+b+r)-b(\beta-\eta) c]}{2 \beta(b+r) c}\right)^{2}-\frac{\Delta(c)}{4 \beta^{2}(b+r)^{2} c^{2}}=0
$$

where

$$
\Delta(c)=[\beta(a+b+r)-b(\beta-\eta) c]^{2}-4 \beta b c(b+r)(a-\beta+\eta)
$$

Proof. From second equation of (3.5.1):

$$
\beta x y-b y-r y+b q y-\frac{a y}{1+c y}=0 \Leftrightarrow y\left(\beta x-b-r+b q-\frac{a}{1+c y}\right)=0
$$

1) $y=0$ then $x=1$ : This is a disease free equilibrium $(1,0)$

2) $y \neq 0(y>0)$ then

$$
\beta x-b-r+b q-\frac{a}{1+c y}=0 \Leftrightarrow \beta x=b+r-b q+\frac{a}{1+c y}
$$

and also imply

$$
x=\frac{b+r-b q}{\beta}+\frac{a}{\beta(1+c y)} .
$$


Substitute those values to the first equation of (3.5.1), we have

$$
\begin{aligned}
& b-\left(b+r-b q+\frac{a}{1+c y}\right) y-b\left[\frac{b+r-b q}{\beta}+\frac{a}{\beta(1+c y)}\right]-b q y=0 \\
& b-\left(b+r+\frac{a}{1+c y}\right) y-b\left[\frac{b+r-b q}{\beta}+\frac{a}{\beta(1+c y)}\right]=0 \\
& b-(b+r) y-\frac{a y}{1+c y}-\frac{b(b+r-b q)}{\beta}-\frac{a b}{\beta(1+c y)}=0 \\
& \beta b(1+c y)-\beta(1+c y)(b+r) y-a \beta y-b(1+c y)(b+r-b q)-a b=0 .
\end{aligned}
$$

Expand and re-arrange the equation above, together with $\eta=b+r-b q$, we end up with equation (3.5.4)

$$
\beta(b+r) c y^{2}+[\beta(b+r+a)-b(\beta-\eta) c] y+b(a-\beta+\eta)=0
$$

3) By completing square on (3.5.4) we get,

$$
\begin{aligned}
& \beta(b+r) c y^{2}+[\beta(b+r+a)-b(\beta-\eta) c] y+b(a-\beta+\eta)=0 \\
& y^{2}+\left[\frac{\beta(b+r+a)-b(\beta-\eta) c}{\beta(b+r) c}\right] y+\frac{b(a-\beta+\eta)}{\beta(b+r) c}=0 \\
& {\left[y+\frac{\beta(b+r+a)-b(\beta-\eta) c}{2 \beta(b+r) c}\right]^{2}-\left[\left(\frac{[\beta(b+r+a)-b(\beta-\eta) c]}{2 \beta(b+r) c}\right)^{2}-\frac{b(a-\beta+\eta)}{\beta(b+r) c}\right]=0}
\end{aligned}
$$

Since

$$
\begin{aligned}
& \left(\frac{[\beta(a+b+r)-b(\beta-\eta) c]}{2 \beta(b+r) c}\right)^{2}-\frac{b(a-\beta+\eta)}{\beta(b+r) c} \\
& =\frac{[\beta(a+b+r)-b(\beta-\eta) c]^{2}-4 \beta b c(b+r)(a-\beta+\eta)}{4 \beta^{2}(b+r)^{2} c^{2}}=\frac{\Delta(c)}{4 \beta^{2}(b+r)^{2} c^{2}}
\end{aligned}
$$

Therefore equation (3.5.2) is equivalent equation (3.5.5) with the value of $\Delta(c)$ defined above. 
Notation: Let

$$
\begin{aligned}
\Delta_{0} & :=4 \beta^{2} b^{2} a(b+r)(\beta+b q)(a-\beta+\eta), \\
c_{0} & :=\frac{\beta(a+b+r)-\sqrt{\Delta(c)}}{b(\beta-\eta)}, \\
c_{1} & :=\frac{\beta b[a(\beta+b q)-(b+r)(\beta-\eta-a)]-\sqrt{\Delta_{0}}}{b^{2}(\beta-\eta)^{2}}, \\
c_{2} & :=\frac{\beta b[a(\beta+b q)-(b+r)(\beta-\eta-a)]+\sqrt{\Delta_{0}}}{b^{2}(\beta-\eta)^{2}} .
\end{aligned}
$$

Lemma 3.5.3. 1) $\Delta_{0}>0$ if and only if $\beta>\eta$ and $a>\beta-\eta$ or $\beta \leq \eta$ and $a>0$.

2) $\Delta_{0}=0$ if and only if $\beta>\eta$ and $a=\beta-\eta$.

3) $\Delta_{0}<0$ if and only if $\beta>\eta$ and $0<a<\beta-\eta$.

Proof. By definition of $\Delta_{0}$ we have

$$
\Delta_{0}:=4 \beta^{2} b^{2} a(b+r)(\beta+b q)(a-\beta+\eta) .
$$

1) If $\beta>\eta$ and $a>\beta-\eta$ or $\beta \leq \eta$ and $a>0$ then $(a-\beta+\eta)>0$ then implies $\Delta_{0}>0$.

2) If $\beta>\eta$ and $a=\beta-\eta$, then $(a+\eta-\beta)=0$, then implies $\Delta_{0}=0$.

3) If $\beta>\eta$ and $0<a<\beta-\eta$ then $(a-\beta+\eta)<0$ then implies $\Delta_{0}<0$. 
Lemma 3.5.4. Assume that $\eta<\beta$ and $\beta-\eta \leq a$ or $\beta \leq \eta$ and $a>0$, then the following assertions hold,

1) $\Delta(c)>0$ if and only if $0<c<c_{1}$ or $c>c_{2}$.

2) $\Delta(c)<0$ if and only if $c_{1}<c<c_{2}$.

3) $\Delta(c)=0$ if and only if $c=c_{1}$ or $c=c_{2}$.

By definition of equation $\Delta(c)$ from (3.5.6), we expand the value of $\Delta(c)$

$$
\begin{aligned}
\Delta(c) & =b^{2}(\beta-\eta)^{2} c^{2}-2 \beta b[(\beta-\eta)(a+b+r)+2(b+r)(a-\beta+\eta)] c+\beta^{2}(a+b+r) \\
& =b^{2}(\beta-\eta)^{2} c^{2}-2 \beta b[(\beta-\eta)(a-b-r)+2 a(b+r)] c+\beta^{2}(a+b+r) \\
& =b^{2}(\beta-\eta)^{2} c^{2}-2 \beta b[a(\beta-\eta)-(\beta-\eta)(b+r)+2 a(b+r)] c+\beta^{2}(a+b+r) \\
& =b^{2}(\beta-\eta)^{2} c^{2}-2 \beta b[a(\beta-\eta+2 b+2 r)-(\beta-\eta)(b+r)] c+\beta^{2}(a+b+r) \\
& =b^{2}(\beta-\eta)^{2} c^{2}-2 \beta b[a(\beta+b q+b+r)-(\beta-\eta)(b+r)] c+\beta^{2}(a+b+r) \\
& =b^{2}(\beta-\eta)^{2} c^{2}-2 \beta b[a(\beta+b q)+a(b+r)-(\beta-\eta)(b+r)] c+\beta^{2}(a+b+r) \\
& =b^{2}(\beta-\eta)^{2} c^{2}-2 \beta b[a(\beta+b q)-(b+r)(\beta-\eta-a)] c+\beta^{2}(a+b+r)
\end{aligned}
$$

therefore

$$
\Delta(c)=b^{2}(\beta-\eta)^{2} c^{2}-2 \beta b[a(\beta+b q)-(b+r)(\beta-\eta-a)] c+\beta^{2}(a+b+r)^{2} .
$$

By completing square of equation (3.5.7)

$$
\begin{aligned}
& \Delta(c)=b^{2}(\beta-\eta)^{2}\left[c-\frac{\beta b[a(\beta+b q)-(b+r)(\beta-\eta-a)]}{b^{2}(\beta-\eta)^{2}}\right]^{2} \\
& +\beta^{2}(a+b+r)^{2}-b^{2}(\beta-\eta)^{2}\left(\frac{\beta b[a(\beta+b q)-(b+r)(\beta-\eta-a)]}{b^{2}(\beta-\eta)^{2}}\right)^{2}
\end{aligned}
$$


however

$$
\begin{array}{r}
{[\beta(a+b+r)]^{2}-\frac{(\beta b[a(\beta+b q)-(b+r)(\beta-\eta-a)])^{2}}{b^{2}(\beta-\eta)^{2}}} \\
=\left[\beta(a+b+r)-\frac{\beta b[a(\beta+b q)-(b+r)(\beta-\eta-a)]}{b(\beta-\eta)}\right] \\
\quad\left[\beta(a+b+r)+\frac{\beta b[a(\beta+b q)-(b+r)(\beta-\eta-a)]}{b(\beta-\eta)}\right]
\end{array}
$$

since

$$
\begin{aligned}
& {\left[\frac{\beta b(a+b+r)(\beta-\eta)-\beta b[a(\beta+b q)-(b+r)(\beta-\eta-a)]}{b(\beta-\eta)}\right]} \\
& =\left[\frac{\beta b[(a+b+r)(\beta-\eta)-[a(\beta+b q)-(b+r)(\beta-\eta-a)]]}{b(\beta-\eta)}\right] \\
& =\frac{\beta b[a(\beta-\eta-\beta-b q-b-r)+2(b+r)(\beta-\eta)]}{b(\beta-\eta)} \\
& =\frac{\beta b[a(b q-b-r-b q-b-r)+2(b+r)(\beta-\eta)]}{b(\beta-\eta)} \\
& =\frac{\beta b[-2 a(b+r)+2(b+r)(\beta-\eta)]}{b(\beta-\eta)}=\frac{2 \beta b(b+r)(\beta-\eta-a)}{b(\beta-\eta)}
\end{aligned}
$$

and

$$
\begin{aligned}
& {\left[\frac{\beta b(a+b+r)(\beta-\eta)+\beta b[a(\beta+b q)-(b+r)(\beta-\eta-a)]}{b(\beta-\eta)}\right]} \\
& =\left[\frac{\beta b[(a+b+r)(\beta-\eta)+[a(\beta+b q)-(b+r)(\beta-\eta-a)]]}{b(\beta-\eta)}\right] \\
& =\frac{\beta b[a(\beta-\eta)+(b+r)(\beta-\eta)+a(\beta+b q)-(b+r)(\beta-\eta)+a(b+r)]}{b(\beta-\eta)} \\
& =\frac{\beta b[a(2 \beta+b q-b-r+b q+b+r)]}{b(\beta-\eta)}=\frac{2 \beta a b(\beta+b q)}{b(\beta-\eta)}
\end{aligned}
$$

therefore

$$
\Delta(c)=b^{2}(\beta-\eta)^{2}\left[c-\frac{\beta b[a(\beta+b q)-(b+r)(\beta-\eta-a)]}{b^{2}(\beta-\eta)^{2}}\right]^{2}-\frac{\Delta_{0}}{b^{2}(\beta-\eta)^{2}} .
$$


Together with lemma (3.5.3) then we have

$$
\begin{aligned}
\Delta(c)=b^{2}(\beta-\eta)^{2}\left(\left[c-\frac{\beta b[a(\beta+b q)-(b+r)(\beta-\eta-a)]}{b^{2}(\beta-\eta)^{2}}\right]^{2}-\frac{\Delta_{0}}{b^{4}(\beta-\eta)^{4}}\right) \\
=b^{2}(\beta-\eta)^{2}\left(\left[c-\frac{\beta b[a(\beta+b q)-(b+r)(\beta-\eta-a)]}{b^{2}(\beta-\eta)^{2}}\right]^{2}-\left[\frac{\sqrt{\Delta_{0}}}{b^{2}(\beta-\eta)^{2}}\right]\right) \\
=b^{2}(\beta-\eta)^{2}\left[c-\frac{\beta b[a(\beta+b q)-(b+r)(\beta-\eta-a)]}{b^{2}(\beta-\eta)^{2}}-\frac{\sqrt{\Delta_{0}}}{b^{2}(\beta-\eta)^{2}}\right] \\
\quad\left[c-\frac{\beta b[a(\beta+b q)-(b+r)(\beta-\eta-a)]}{b^{2}(\beta-\eta)^{2}}+\frac{\sqrt{\Delta_{0}}}{b^{2}(\beta-\eta)^{2}}\right] \\
=b^{2}(\beta-\eta)^{2}\left[c-\frac{\beta b[a(\beta+b q)-(b+r)(\beta-\eta-a)]-\sqrt{\Delta_{0}}}{b^{2}(\beta-\eta)^{2}}\right] \\
\\
{\left[c-\frac{\beta b[a(\beta+b q)-(b+r)(\beta-\eta-a)]+\sqrt{\Delta_{0}}}{b^{2}(\beta-\eta)^{2}}\right] }
\end{aligned}
$$

thus

$$
\Delta(c)=b^{2}(\beta-\eta)^{2}\left(c-c_{1}\right)\left(c-c_{2} .\right)
$$

Under the condition $\eta<\beta$ and $\beta-\eta \leq a$ or $\beta \leq \eta$ and $a>0$ it is easy to verify that $0<c_{1}<c_{2}$. This together with lemma (3.5.4) imply the results.

Lemma 3.5.5. Assume that $a, b, c, \beta, r>0, q \in[0,1]$. If either $r \leq \beta<b+r$ and $0 \leq q \leq q_{1}$ or $0<\beta<r$ and $0 \leq q \leq 1$, then system (3.3.3) has no positive equilibria.

Proof. Under the condition $r \leq \beta<b+r$ and $0 \leq q \leq q_{1}$ or $0<\beta<r$ and $0 \leq q \leq 1$ we have by lemma (3.5.1) (1), $\beta \leq \eta$. By equation (3.5.4) and the fact that $\beta \leq \eta$ we have

$$
\beta c(b+r) y^{2}-[b c(\beta-\eta)-\beta(b+r+a)] y+b(a+\eta-\beta)>b(a+\eta-\beta)>0 .
$$


Thus no value of $y>0$ can satisfy equation (3.5.4). So that the system (3.3.3) has no positive equilibrium points.

We now give the main result of this section.

Theorem 3.5.6. Assume that $0<\eta<\beta$ and $0<\beta-\eta \leq a$ then the following assertions hold

1) If $c>c_{2}$, then system (3.3.3) has two positive interior equilibria $\left(x_{1}, y_{1}\right)$ and $\left(x_{2}, y_{2}\right)$ where

$$
y_{1}=\frac{b c(\beta-\eta)-\beta(b+r+a)-\sqrt{\Delta(c)}}{2 \beta c(b+r)}, y_{2}=\frac{b c(\beta-\eta)-\beta(b+r+a)+\sqrt{\Delta(c)}}{2 \beta c(b+r)}
$$

and

$$
x_{1}=\frac{\eta\left(1+c y_{1}\right)+a}{\beta\left(1+c y_{1}\right)}, \quad x_{2}=\frac{\eta\left(1+c y_{2}\right)+a}{\beta\left(1+c y_{2}\right)} .
$$

(2) If $c=c_{2}$ then system (3.3.3) has one positive interior equilibrium $\left(x^{*}, y^{*}\right)$ where,

$$
x^{*}=\frac{\eta\left(1+c y^{*}\right)+a}{\beta\left(1+c y^{*}\right)}, y^{*}=\frac{b c(\beta-\eta)-\beta(b+r+a)}{2 \beta c(b+r)} .
$$

(3) If $0<c<c_{2}$ then $(1,0)$ is a positive disease free equilibrium of system (3.3.3).

Proof. By lemma (3.5.2) (2) we have that if $(x, y)$ is an equilibrium point of system (3.3.3) then $y$ is a solution of equation (3.5.2). It is easy to verify that $y_{1}$ and $y_{2}$ given in (3.5.10) are the only solution of (3.5.4). Substituting 
$y_{1}$ and $y_{2}$ into the second equation of (3.5.1) and solving for $x$ give $x_{1}$ and $x_{2}$ respectively. Moreover by definition of $c_{2}$ and $c_{0}$ we have

$$
\begin{aligned}
c_{2}-c_{0} & =\left(\frac{\beta b[a(\beta+b q)-(b+r)(\beta-\eta-a)]+\sqrt{\Delta_{0}}}{b^{2}(\beta-\eta)^{2}}\right)-\left(\frac{\beta(b+r+a)-\sqrt{\Delta(c)}}{b(\beta-\eta)}\right) \\
& =\frac{\beta a b(\beta+b q+b+r-\beta+\eta)-2 \beta b(b+r)(\beta-\eta)+\sqrt{\Delta_{0}}+b(\beta-\eta) \sqrt{\Delta(c)}}{b^{2}(\beta-\eta)^{2}} \\
& =\frac{\beta a b(b q+b+r+b+r-b q)-2 \beta b(b+r)(\beta-\eta)+\sqrt{\Delta_{0}}+b(\beta-\eta) \sqrt{\Delta(c)}}{b^{2}(\beta-\eta)^{2}} \\
& =\frac{2 \beta a b(b+r)-2 \beta b(b+r)(\beta-\eta)+\sqrt{\Delta_{0}}+b(\beta-\eta) \sqrt{\Delta(c)}}{b^{2}(\beta-\eta)^{2}} \\
& =\frac{2 \beta b(b+r)(a-\beta+\eta)+\sqrt{\Delta_{0}}+b(\beta-\eta) \sqrt{\Delta(c)}}{b^{2}(\beta-\eta)^{2}}
\end{aligned}
$$

so that

$$
c_{2}-c_{0}=\frac{2 \beta b(b+r)(a-\beta+\eta)+\sqrt{\Delta_{0}}+b(\beta-\eta) \sqrt{\Delta(c)}}{b^{2}(\beta-\eta)^{2}}
$$

By (3.5.10) we have

$y_{1}=\frac{[b(\beta-\eta) c-\beta(a+b+r)]-\sqrt{\Delta(c)}}{2 \beta(b+r) c}=\frac{b(\beta-\eta)\left[c-\frac{(\beta(a+b+r)+\sqrt{\Delta(c)})}{b(\beta-\eta)}\right]}{2 \beta(b+r) c}$

or

$$
y_{1}=\frac{b(\beta-\eta)\left(c-c_{0}\right)}{2 \beta(b+r) c} .
$$

1)If $c>c_{2}$ then by Lemma (3.5.4) (1) and Theorem (3.5.6) (1), $y_{1}, y_{2}$ are solutions of equation (3.5.4).

Since $c>c_{2}$ then $c-c_{0}>c_{2}-c_{0}>0$ because $\beta>\eta$ and $a \geq \beta-\eta$. The result follows from (3.5.13). Therefore $y_{1}>0$ implies $y_{2}>0$ since $y_{1}<y_{2}$. 
Hence $\left(x_{1}, y_{1}\right)$ and $\left(x_{2}, y_{2}\right)$ are the two positive equilibria of system (3.3.3).

2) If $c=c_{2}$ then by Lemma (3.5.4) we have $\Delta(c)=0$ and by (3.5.11) we have

$$
y_{1}=y_{2}=y^{*}=\frac{b(\beta-\eta) c_{2}-\beta(a+b+r)}{2 \beta(b+r) c_{2}}=\frac{b(\beta-\eta)\left[c_{2}-\frac{\beta(a+b+r)}{b(\beta-\eta)}\right]}{2 \beta(b+r) c_{2}} .
$$

Now

$$
\begin{aligned}
& c_{2}-\frac{\beta(a+b+r)}{b(\beta-\eta)}=\frac{\beta b[a(\beta+b q)-(b+r)(\beta-\eta-a)]+\sqrt{\Delta_{0}}}{b^{2}(\beta-\eta)^{2}}-\frac{\beta(a+b+r)}{b(\beta-\eta)} \\
& =\frac{\beta b[a(\beta+b q)-(b+r)(\beta-\eta-a)]-\beta b(\beta-\eta)(a+b+r)+\sqrt{\Delta_{0}}}{b^{2}(\beta-\eta)^{2}} \\
& =\frac{\beta b[a(\beta+b q)-(b+r)(\beta-\eta-a)-(\beta-\eta)(a+b+r)]+\sqrt{\Delta_{0}}}{b^{2}(\beta-\eta)^{2}} \\
& =\frac{\beta b[a(\beta+b q)-2(b+r)(\beta-\eta)+a(b+r)-a(\beta-\eta)]+\sqrt{\Delta_{0}}}{b^{2}(\beta-\eta)^{2}} \\
& =\frac{\beta b[a(\beta+b q+b+r-\beta+\eta)-2(b+r)(\beta-\eta)]+\sqrt{\Delta_{0}}}{b^{2}(\beta-\eta)^{2}} \\
& =\frac{\beta b[2 a(b+r)-2(b+r)(\beta-\eta)]+\sqrt{\Delta_{0}}}{b^{2}(\beta-\eta)^{2}} \\
& =\frac{2 \beta b(b+r)(a-\beta+\eta)+\sqrt{\Delta_{0}}}{b^{2}(\beta-\eta)^{2}}
\end{aligned}
$$

or

$$
c_{2}-\frac{\beta(a+b+r)}{b(\beta-\eta)}=\frac{2 \beta b(b+r)(a-\beta+\eta)+\sqrt{\Delta_{0}}}{b^{2}(\beta-\eta)^{2}}
$$

Hence

$$
y_{1}=y_{2}=y^{*}=\frac{b(\beta-\eta) c_{2}-\beta(a+b+r)}{2 \beta(b+r) c_{2}}>0
$$

since $\beta>\eta$ and $a>\beta-\eta$.

Substituting $y^{*}$ into the second equation of (3.5.1) and solving for $x$ gives $x^{*}$.

Hence system (3.3.3) has one positive equilibrium point $\left(x^{*}, y^{*}\right)$. 
3) We consider the following 3 cases:

i) If $c_{1}<c<c_{2}$ then by Lemma (3.5.4) (2), $\Delta(c)<0$ so that equation (3.5.4) has no solutions. Hence system (3.3.3) has no positive interior equilibria.

ii) If $c=c_{1}$ then by Lemma (3.5.4) (3), $\Delta(c)=0$ and by (3.5.11), we have

$$
y_{1}=y_{2}=y^{*}=\frac{b(\beta-\eta) c_{1}-\beta(a+b+r)}{2 \beta(b+r) c_{1}}=\frac{b(\beta-\eta)\left[c_{1}-\frac{\beta(a+b+r)}{b(\beta-\eta)}\right]}{2 \beta(b+r) c_{1}} .
$$

Since

$$
c_{1}-\frac{\beta(a+b+r)}{b(\beta-\eta)}=\frac{2 \beta b(b+r)(a-\beta+\eta)-\sqrt{\Delta_{0}}}{b^{2}(\beta-\eta)^{2}}
$$

and

$$
\begin{aligned}
c_{1}- & \frac{\beta(a+b+r)}{b(\beta-\eta)}=\frac{2 \beta b(b+r)(a-\beta+\eta)-\sqrt{\Delta_{0}}}{b^{2}(\beta-\eta)^{2}} \\
= & \frac{[2 \beta b(b+r)(a-\beta+\eta)]^{2}-\Delta_{0}}{b^{2}(\beta-\eta)^{2}\left[2 \beta b(b+r)(a-\beta+\eta)+\sqrt{\Delta_{0}}\right]} \\
= & \frac{[2 \beta b(b+r)(a-\beta+\eta)]^{2}-4 \beta^{2} b^{2} a(b+r)(\beta+b q)(a-\beta+\eta)}{b^{2}(\beta-\eta)^{2}\left[2 \beta b(b+r)(a-\beta+\eta)+\sqrt{\Delta_{0}}\right]} \\
= & \frac{4 \beta^{2} b^{2}(b+r)(a-\beta+\eta)[(b+r)(a-\beta+\eta)-a(\beta+b q)]}{b^{2}(\beta-\eta)^{2}\left[2 \beta b(b+r)(a-\beta+\eta)+\sqrt{\Delta_{0}}\right]} \\
= & \frac{4 \beta^{2} b^{2}(b+r)(a-\beta+\eta)[a(b+r-\beta-b q)+(b+r)(\eta-\beta)]}{b^{2}(\beta-\eta)^{2}\left[2 \beta b(b+r)(a-\beta+\eta)+\sqrt{\Delta_{0}}\right]} \\
= & \frac{4 \beta^{2} b^{2}(b+r)(a-\beta+\eta)[a(\eta-\beta)+(b+r)(\eta-\beta)]}{b^{2}(\beta-\eta)^{2}\left[2 \beta b(b+r)(a-\beta+\eta)+\sqrt{\Delta_{0}}\right]} \\
= & \frac{4 \beta^{2} b^{2}(b+r)(a-\beta+\eta)(\eta-\beta)(a+b+r)}{b^{2}(\beta-\eta)^{2}\left[2 \beta b(b+r)(a-\beta+\eta)+\sqrt{\Delta_{0}}\right]}
\end{aligned}
$$


so that

$$
c_{1}-\frac{\beta(a+b+r)}{b(\beta-\eta)}=\frac{4 \beta^{2} b^{2}(b+r)(a-\beta+\eta)(\eta-\beta)(a+b+r)}{b^{2}(\beta-\eta)^{2}\left[2 \beta b(b+r)(a-\beta+\eta)+\sqrt{\Delta_{0}}\right]} .
$$

Hence $y_{1}=y_{2}=y^{*}<0$ because $\beta>\eta$ and $a \geq \beta-\eta$.

iii) If $0<c<c_{1}$ then by Lemma (3.5.4) (1) $\Delta(c)>0$, then equation (3.5.4) has 2 solutions indicated at (3.5.10).

By $(3.5 .10)$

$$
\begin{aligned}
y_{2} & =\frac{b(\beta-\eta) c-\beta(a+b+r)+\sqrt{\Delta(c)}}{2 \beta(b+r) c} \\
& =-\frac{\beta(a+b+r)-b(\beta-\eta) c-\sqrt{\Delta(c)}}{2 \beta(b+r) c} \\
& =-\frac{[\beta(a+b+r)-b(\beta-\eta) c]^{2}-\Delta(c)}{2 \beta(b+r) c[\beta(a+b+r)-b(\beta-\eta) c+\sqrt{\Delta(c)}]} .
\end{aligned}
$$

By definition of $\Delta(c)$ from (3.5.3) we obtain

$$
y_{2}=-\frac{4 \beta b c(b+r)(a-\beta+\eta)}{2 \beta(b+r) c[\beta(a+b+r)-b(\beta-\eta) c+\sqrt{\Delta(c)}]} .
$$

We also have

$$
\beta(a+b+r)-b(\beta-\eta) c+\sqrt{\Delta(c)}>\beta(a+b+r)-b(\beta-\eta) c
$$

but

$$
\beta(a+b+r)-b(\beta-\eta) c=b(\beta-\eta)\left(\frac{\beta(a+b+r)}{b(\beta-\eta)}-c\right) .
$$

Since $c<c_{1} \Leftrightarrow-c>-c_{1}$ so

$$
\frac{\beta(a+b+r)}{b(\beta-\eta)}-c>\frac{\beta(a+b+r)}{b(\beta-\eta)}-c_{1} .
$$


From the result (3.5.13) we imply

$$
\frac{\beta(a+b+r)}{b(\beta-\eta)}-c>\frac{\beta(a+b+r)}{b(\beta-\eta)}-c_{1}>0 .
$$

From (3.5.14) to (3.5.16) we have

$$
\beta(a+b+r)-b(\beta-\eta) c+\sqrt{\Delta(c)}>0
$$

or

$$
y_{2}=\frac{b(\beta-\eta) c-\beta(a+b+r)+\sqrt{\Delta(c)}}{2 \beta(b+r) c}<0 .
$$

Since $y_{1}<y_{2}$ and $y_{2}<0$ so that $y_{1}<0$. Hence systems (3.3.3) has no positive interior equilibrium. 


\section{Chapter 4}

\section{Qualitative behaviour analysis of the equilibria points}

From theorem (3.5.6) we have total 3 interior equilibrium points. We will study the phase portrait and stability of each interior equilibrium point.

Let $A(x, y)$ be the Jacobian matrix of $f$ and $g$ defined in the system (3.3.3). By (2.1.4) and (3.3.3) we have

$$
A(x, y)=\left(\begin{array}{cc}
-\beta y-b & -\beta x-q b \\
\beta y & \beta x-\eta-\frac{a}{(1+c y)^{2}}
\end{array}\right)
$$

Together with definition of $\eta=b+r-b q$ then we have

$$
|A(x, y)|=\frac{\beta y}{(1+c y)^{2}}\left[(1+c y)^{2}(b+r)+a\right]-\beta b x+b\left[\eta+\frac{a}{(1+c y)^{2}}\right]
$$

and

$$
\operatorname{tr}(A(x, y))=\frac{\beta(x-y)(1+c y)^{2}-\left[(b+\eta)(1+c y)^{2}+a\right]}{(1+c y)^{2}} .
$$


Also, for $y>0$, from the second equation of system (3.5.1) we have

$$
\beta x-b-r+b q-\frac{a}{1+c y}=0 \Leftrightarrow-\beta x-b q=-\frac{a}{1+c y}-b-r
$$

and $\quad \beta x-\eta=\frac{a}{1+c y}$. Substitute those values above into (4.0.1), we have

$$
\mathrm{A}(x, y)=\left(\begin{array}{cc}
-\beta y-b & -\frac{a}{1+c y}-b-r \\
\beta y & \frac{a}{1+c y}-\frac{a}{(1+c y)^{2}}
\end{array}\right)=\left(\begin{array}{cc}
-\beta y-b & -\frac{a}{1+c y}-b-r \\
\beta y & \frac{a c y}{(1+c y)^{2}}
\end{array}\right)
$$

therefore for $y>0$

$$
|\mathrm{A}(x, y)|=(-\beta y-b) \frac{a c y}{(1+c y)^{2}}+\beta y\left(\frac{a}{1+c y}+b+r\right)
$$

and

$$
\operatorname{tr}(\mathrm{A}(\mathrm{x}, \mathrm{y}))=(-\beta y-b)+\frac{a c y}{(1+c y)^{2}} .
$$

Now we can use the $|\mathrm{A}(x, y)|$ and the $\operatorname{tr}(\mathrm{A}(x, y))$ from (4.0.2) - (4.0.5) depends on particular cases.

We first consider the properties of the disease-free equilibrium $(1,0)$ of (3.3.3).

Theorem 4.0.1. (1) If $0<\eta<\beta$ and $0<a<\beta-\eta$, then $(1,0)$ is a saddle of the system (3.3.3).

(2) If either $0<\beta<\eta$ and $a>0$ or $\eta \leq \beta<\eta+b$ and $a>\beta-\eta$, then $(1,0)$ is a stable node of the system (3.3.3). Moreover, $(1,0)$ of the system (3.3.3) is globally asymptotically stable.

(3) If $\eta \leq \beta<\eta+b, a=\beta-\eta$ and $c \neq \frac{\beta}{\beta-\eta}$ then $(1,0)$ is a saddle-node of the system (3.3.3).

Proof. Substitute $(x, y)=(1,0)$ to (4.0.2) and (4.0.3), we obtain

$$
|A(1,0)|=b(a+\eta-\beta)
$$


and

$$
\operatorname{tr}(A(1,0))=\beta-\eta-a-b
$$

1) By (4.0.6), we have $|A(1,0)|<0$. The result follows from Lemma (2.1.2) $(i)$. Hence $(x, y)=(1,0)$ is a saddle of $(3.3 .3)$.

2) Under each of the conditions $(i)$ and $(i i)$ of theorem (4.0.1) (2), then $|A(1,0)|>0$ and $\operatorname{tr}(A(1,0))<0$.

We also have

$$
\begin{aligned}
\operatorname{tr}(A(1,0))^{2}-4|A(1,0)| & =(\beta-\eta-a-b)^{2}-4 b(a+\eta-\beta) \\
& =(\beta-\eta-a)^{2}-2 b(\beta-\eta-a)+b^{2}+4 b(\beta-a-\eta) \\
& =(\beta-\eta-a)^{2}+2 b(\beta-\eta-a)+b^{2} \\
& =(\beta-\eta-a+b)^{2} \geq 0 .
\end{aligned}
$$

The first result follows from Lemma $(2.1 .2)(i i)$. Hence $(x, y)=(1,0)$ is a stable node of (3.3.3).

In order to prove $(1,0)$ of system (3.3.3) is globally asymptotically stable, we need apply the Lemma (2.1.4). This lemma is a special case of the wellknown Poincare'-Bendixson Trichotomy theorem.

Let $B=\left\{(u, v) \in \mathbb{R}_{+}^{2}: u+v \leq 1\right\}$. Then $B$ is a bounded closed subset of $\mathbb{R}^{2}$. We can see that the equilibrium point $(1,0)$ of $(3.3 .3)$ is in $B$. Since $(1,0)$ is on the boundary of $B$ then every positive solution of (3.3.3) converges to $(1,0)$ as $t \rightarrow \infty$. Therefore, $(1,0)$ of $(3.3 .3)$ is globally asymptotically stable. 
3) Since $a=\beta-\eta$, by (4.0.6) and (4.0.7) we have $|A(1,0)|=0$ and $\operatorname{tr}(A(1,0))=-b<0$. We change the equilibrium point $(1,0)$ to the origin $(0,0)$ by the change of variables $u_{1}=x-1$ and $v_{1}=y$. Note that $a=\beta-\eta$. Then system (3.3.3) becomes

$$
\left\{\begin{array}{l}
\dot{u}_{1}=\dot{x}=b-\beta\left(u_{1}+1\right) v_{1}-b\left(u_{1}+1\right)-q b v_{1}=-\beta u_{1} v_{1}-b\left[\frac{(\beta+q b)}{b} v_{1}+u_{1}\right] \\
\dot{v}_{1}=\dot{y}=\beta\left(u_{1}+1\right) v_{1}-\eta v_{1}-\frac{a v_{1}}{1+c v_{1}}=\beta u_{1} v_{1}+\frac{(\beta-\eta) c v_{1}^{2}}{1+c v_{1}}
\end{array}\right.
$$

Let $\delta=(\beta+q b) b^{-1}, u_{2}=u_{1}+\delta v_{1}$ and $v_{2}=v_{1}$, then the last system becomes

$$
\begin{aligned}
\dot{u}_{2} & =\dot{u}_{1}+\delta \dot{v}_{1}=-\beta u_{1} v_{1}-b\left[\frac{(\beta+q b)}{b} v_{1}+u_{1}\right]+\delta \beta u_{1} v_{1}+\frac{(\beta-\eta) \delta c v_{1}^{2}}{1+c v_{1}} \\
& =(\delta-1) \beta u_{1} v_{1}-b\left[\frac{(\beta+q b)}{b} v_{1}+u_{1}\right]+\frac{(\beta-\eta) \delta c v_{1}^{2}}{1+c v_{1}} \\
& =(\delta-1) \beta\left[u_{2} v_{2}-\delta v_{2}^{2}\right]-b u_{2}+\frac{(\beta-\eta) \delta c v_{2}^{2}}{1+c v_{2}} \\
& =-\delta \beta(\delta-1) v_{2}^{2}+(\delta-1) \beta u_{2} v_{2}+\frac{(\beta-\eta) \delta c v_{2}^{2}}{1+c v_{2}}-b u_{2}
\end{aligned}
$$

and

$$
\dot{v}_{2}=\beta v_{2}\left[u_{2}-\delta v_{2}\right]+\frac{(\beta-\eta) \delta c v_{2}^{2}}{1+c v_{2}}=\beta v_{2} u_{2}-\frac{[(\beta-\eta) \delta c-\beta \delta] v_{2}^{2}-\beta \delta c v_{2}^{3}}{1+c v_{2}}
$$

Let $u=v_{2}$ and $v=u_{2}$. Then the above last two equations become,

$$
\left\{\begin{array}{l}
\dot{u}=p(u, v) \\
\dot{v}=-b v+q(u, v)
\end{array}\right.
$$

where

$p(u, v)=-[(\beta-\eta) \delta c-\beta \delta] u^{2}+\beta u v+\beta c \delta u^{3}-\left[[(\beta-\eta) \delta c-\beta \delta] u_{2}^{2}-\beta \delta c u^{3}\right] \sum_{n=1}^{\infty} \frac{(-1)^{n}}{n !}(c u)^{n}$ 
and

$$
q(u, v)=-\delta(\delta-1) \beta u^{2}+(\delta-1) \beta u v+(\beta-\eta) \delta c u^{2} \sum_{n=1}^{\infty} \frac{(-1)^{n}}{n !}(c u)^{n}
$$

Since $\varrho:=-b \neq 0$ and $\left.a_{20}:=-[(\beta-\eta) \delta c-\beta \delta)\right] \neq 0$ provided $c \neq \frac{\beta}{\beta-\eta}$. It follows from lemma $(2.1 .3)$ that $(1,0)$ is a saddle-node of system (3.3.3).

Lemma 4.0.2. Assume that $a, b, c, r>0, q \in[0,1], 0<\eta<\beta$ and $a>\beta-\eta$ then

1) The equation (4.0.4) is equivalent to

$$
|\mathrm{A}(x, y)|=\frac{y}{(1+c y)^{2}}\left[\beta(b+r) y^{2} c^{2}+[2 \beta(b+r) y-a b] c+\beta(a+b+r)\right] .
$$

2) If $c=c_{2}$ then $\left|\mathrm{A}\left(x^{*}, y^{*}\right)\right|=0$.

3) If $c>c_{2}$ then $\left|\mathrm{A}\left(x_{2}, y_{2}\right)\right|>0$.

Proof.

$$
\begin{aligned}
|\mathrm{A}(x, y)| & =(-\beta y-b) \frac{a c y}{(1+c y)^{2}}+\beta y\left(\frac{a}{1+c y}+b+r\right) \\
& =y\left[\beta\left(\frac{a}{1+c y}+b+r\right)-(\beta y+b) \frac{a c}{(1+c y)^{2}}\right] \\
& =y\left[\frac{a \beta}{1+c y}+\beta(b+r)-\frac{\beta a c y}{(1+c y)^{2}}-\frac{a b c}{(1+c y)^{2}}\right] \\
& =y\left[\frac{a \beta}{1+c y}\left(1-\frac{c y}{1+c y}\right)+\beta(b+r)-\frac{a b c}{(1+c y)^{2}}\right] \\
& =y\left[\frac{a \beta}{(1+c y)^{2}}+\beta(b+r)-\frac{a b c}{(1+c y)^{2}}\right] \\
& =\frac{y}{(1+c y)^{2}}\left[\beta(b+r)(1+c y)^{2}+a(\beta-b c)\right] .
\end{aligned}
$$


Expand and re-arrange the term inside bracket then we obtain

$$
|\mathrm{A}(x, y)|=\frac{y}{(1+c y)^{2}}\left[\beta(b+r) y^{2} c^{2}+[2 \beta(b+r) y-a b] c+\beta(a+b+r)\right] .
$$

2) Substitute the value of $y^{*}$ from (3.5.11) into equation (4.0.8), we have

$$
\left|\mathrm{A}\left(x^{*}, y^{*}\right)\right|=\frac{y^{*}}{\left(1+c y^{*}\right)^{2}}\left[\beta(b+r) y^{* 2} c^{2}+\left[2 \beta(b+r) y^{*}-a b\right] c+\beta(a+b+r)\right] .
$$

Since $c=c_{2}$ then from Lemma (3.5.4) (3) we have $\Delta(c)=0$.

By definition of $\Delta(c)$ from equation (3.5.6) then

$$
[b c(\beta-\eta)-\beta(b+r+a)]^{2}=4 \beta b c(b+r)(a-\beta+\eta)
$$

since

$$
\begin{aligned}
& \beta(b+r) y^{* 2} c^{2}+\left[2 \beta(b+r) y^{*}-a b\right] c+\beta(a+b+r) \\
& =\frac{[b c(\beta-\eta)-\beta(b+r+a)]^{2}}{4 \beta(b+r)}+b c(\beta-\eta)-a b c \\
& =\frac{4 \beta b c(b+r)(a-\beta+\eta)}{4 \beta(b+r)}+b c(\beta-\eta)-a b c \\
& =b c(a-\beta+\eta)+b c(\beta-\eta)-a b c=0
\end{aligned}
$$

therefore $\left|\mathrm{A}\left(x^{*}, y^{*}\right)\right|=0$.

3) From the result of Lemma (4.0.2) (1), we have

$$
\left|\mathrm{A}\left(x_{2}, y_{2}\right)\right|=\frac{y_{2}}{\left(1+c y_{2}\right)^{2}}\left[\beta(b+r) y_{2}{ }^{2} c^{2}+\left[2 \beta(b+r) y_{2}-a b\right] c+\beta(a+b+r)\right]
$$

Since $c>c_{2}$ then $\left(x_{2}, y_{2}\right)$ is a positive equilibria of the system (3.3.3) by theorem (3.5.6). 
Let

$$
\phi\left(a, b, c, r, \beta, y_{2}\right)=\beta(b+r) y_{2}{ }^{2} c^{2}+\left[2 \beta(b+r) y_{2}-a b\right] c+\beta(a+b+r)
$$

Substitute $y_{2}$ from (3.5.7) into $\phi$ defined above then we have

$$
\begin{aligned}
\phi & =\beta(b+r)\left[\frac{b(\beta-\eta) c-\beta(a+b+r)+\sqrt{\Delta(c)}}{2 \beta c(b+r)}\right]^{2} c^{2} \\
& +\beta(a+b+r)+\left(2 \beta(b+r)\left[\frac{b(\beta-\eta) c-\beta(a+b+r)+\sqrt{\Delta(c)}}{2 \beta c(b+r)}\right]-a b\right) c \\
& =\frac{[b(\beta-\eta) c-\beta(a+b+r)+\sqrt{\Delta(c)}]^{2}}{4 \beta(b+r)}+\beta(a+b+r)-a b c \\
& +[b(\beta-\eta) c-\beta(a+b+r)+\sqrt{\Delta(c)}] \\
& =\frac{[b(\beta-\eta) c-\beta(a+b+r)+\sqrt{\Delta(c)}]^{2}}{4 \beta(b+r)}+b(\beta-\eta) c-a b c+\sqrt{\Delta(c)}
\end{aligned}
$$

since

$$
\Delta(c)=[b(\beta-\eta) c-\beta(a+b+r)]^{2}-4 \beta b c(b+r)(a-\beta+\eta)
$$

then

$$
\begin{aligned}
& {[b(\beta-\eta) c-\beta(a+b+r)+\sqrt{\Delta(c)}]^{2}=([b(\beta-\eta) c-\beta(a+b+r)]+\sqrt{\Delta(c)})^{2}} \\
& =[b(\beta-\eta) c-\beta(a+b+r)]^{2}+2[b(\beta-\eta) c-\beta(a+b+r)] \sqrt{\Delta(c)}+\Delta(c) \\
& =2 \Delta(c)+4 \beta b c(b+r)(a-\beta+\eta)+2[b(\beta-\eta) c-\beta(a+b+r)] \sqrt{\Delta(c)}
\end{aligned}
$$


therefore

$$
\begin{aligned}
\phi & =\beta(a+b+r)-a b c+[b(\beta-\eta) c-\beta(a+b+r)+\sqrt{\Delta(c)}] \\
& +\frac{2 \Delta(c)+4 \beta b c(b+r)(a-\beta+\eta)+2[b(\beta-\eta) c-\beta(a+b+r)] \sqrt{\Delta(c)}}{4 \beta(b+r)} \\
& =\frac{2 \sqrt{\Delta(c)}[\sqrt{\Delta(c)}+[b(\beta-\eta) c-\beta(a+b+r)]]}{4 \beta(b+r)} \\
& +b(\beta-\eta) c+\sqrt{\Delta(c)}-a b c+b c(a-\beta+\eta) \\
& =\frac{\sqrt{\Delta(c)}[\sqrt{\Delta(c)}+[b(\beta-\eta) c-\beta(a+b+r)]]}{2 \beta(b+r)}+\sqrt{\Delta(c)} \\
& =\sqrt{\Delta(c)}\left[\frac{\sqrt{\Delta(c)}+[b(\beta-\eta) c-\beta(a+b+r)]}{2 \beta(b+r)}+1\right] \\
& \theta\left(a, b, c, r, \beta, y_{1}\right)=\sqrt{\Delta(c)}\left(c y_{1}+1\right)>0 .
\end{aligned}
$$

Hence

$$
\left|\mathrm{A}\left(x_{2}, y_{2}\right)\right|=\frac{\phi y_{2}}{\left(1+c y_{2}\right)^{2}}>0
$$

since $y_{2}, \phi>0$.

Lemma 4.0.3. If $\beta>\eta$, and $c=c_{2}$ then there exists $a_{0} \in(\beta-\eta, \infty)$ such that $\operatorname{tr}\left(A\left(x^{*}, y^{*}\right)\right)<0$ for $a \in\left[\beta-\eta, a_{0}\right)$

Proof. Under the condition $c=c_{2}$, by lemma (??) (3) we have $\Delta(c)=0$. Moreover by (3.5.8) we have $y_{1}=y_{2}=y^{*}$. By the formula of $y^{*}$ and $c_{2}$ we have, 


$$
\begin{aligned}
c_{2} y^{*} & =\frac{b c(\beta-\eta)-\beta(b+r+a)}{2 \beta(b+r)} \\
& =\frac{\beta b[(\beta-\eta)(b+r+a)+2(b+r)(a+\eta-\beta)]+\sqrt{\Delta_{0}}-\beta b(\beta-\eta)(b+r+a)}{2 \beta b(b+r)(\beta-\eta)} \\
& =\frac{2 \beta b(b+r)(a+\eta-\beta)+\sqrt{\Delta_{0}}}{2 \beta b(b+r)(\beta-\eta)}
\end{aligned}
$$

then

$$
\left(1+c_{2} y^{*}\right)=\frac{2 \beta b(b+r) a+\sqrt{\Delta_{0}}}{2 \beta b(b+r)(\beta-\eta)} .
$$

By (3.5.11) and (4.0.5) with $(x, y)=\left(x^{*}, y^{*}\right)$ we have,

$$
\operatorname{tr}\left(A\left(x^{*}, y^{*}\right)\right)=\frac{-\left(\beta y^{*}+b\right)\left(1+c_{2} y^{*}\right)^{2}+a c_{2} y^{*}}{\left(1+c_{2} y^{*}\right)^{2}}
$$

Substituting, $a=\beta-\eta$, we have by (4.0.10) $\left(1+c_{2} y^{*}\right)^{2}=1$, thus $c_{2} y^{*}=0$ and the above equation becomes

$$
\operatorname{tr}\left(A\left(x^{*}, y^{*}\right)=-\left(\beta y^{*}+b\right)\right.
$$

It follows from the continuity of $\operatorname{tr}\left(A\left(x^{*}, y^{*}\right)\right)$ that there exists an $a_{0} \in(\beta-$ $\eta, \infty)$ such that $\operatorname{tr}\left(A\left(x^{*}, y^{*}\right)\right)<0$ for $a \in[\beta-\eta, \infty)$.

Theorem 4.0.4. If $\beta>\eta$ and $c=c_{2}$, then there exists an $a_{0} \in(\beta-\eta, \infty)$ such that the equilibrium point $\left(x^{*}, y^{*}\right)$ is a saddle node of (3.3.3) for $a \in$ $\left[\beta-\eta, a_{0}\right)$.

Proof. By lemma (4.0.2) (2) and (4.0.11) we have that $\left|A\left(x^{*}, y^{*}\right)\right|=0$ and $\operatorname{tr}\left(A\left(x^{*}, y^{*}\right)\right) \neq 0$ respectively. We change the equilibrium point $\left(x^{*}, y^{*}\right)$ to 
the origin by the change of variables, $u_{1}=x-x^{*}$ and $v_{1}=y-y^{*}$, then system (3.3.3) becomes

$$
\begin{aligned}
\dot{u}_{1} & =b-\beta\left(u_{1}+x^{*}\right)\left(v_{1}+y^{*}\right)-b\left(u_{1}+x^{*}\right)-q b\left(v_{1}+y^{*}\right) \\
& =-\beta u_{1} v_{1}-\left(\beta y^{*}+b\right) u_{1}-\left(\beta x^{*}+q b\right) v_{1}-\left(\beta x^{*} y^{*}+b x^{*}+q b y^{*}-b\right) \\
& =-\beta u_{1} v_{1}-\left(\beta y^{*}+b\right) u_{1}-\left(\beta x^{*}+q b\right) v_{1}
\end{aligned}
$$

and

$$
\begin{aligned}
\dot{v}_{1}= & \beta u_{1} v_{1}+\beta u_{1} y^{*}+\beta v_{1} x^{*}+\beta x^{*} y^{*}-\eta v_{1}+\beta v_{1} x^{*}-\eta v_{1}-\eta y^{*}-\frac{a\left(v_{1}+y^{*}\right)}{1+c\left(v_{1}+y^{*}\right)} \\
= & \beta u_{1} v_{1}+\beta u_{1} y^{*}+\beta v_{1} x^{*}-\eta v_{1}-\eta y^{*}+\frac{\left[\eta\left(1+c y^{*}\right)+a\right] y^{*}}{1+c y^{*}}-\frac{a\left(v_{1}+y^{*}\right)}{1+c\left(v_{1}+y^{*}\right)} \\
= & \beta u_{1} v_{1}+\beta u_{1} y^{*}+\beta v_{1} x^{*}-\eta v_{1}-\frac{a v_{1}}{\left(1+c y^{*}\right)\left[1+c\left(v_{1}+y^{*}\right)\right]} \\
= & \beta u_{1} v_{1}+\beta u_{1} y^{*}+\frac{\left(\beta x^{*}-\eta\right)\left(1+c y^{*}\right) c v_{1}^{2}+\left(\beta x^{*}-\eta\right)\left(1+c y^{*}\right)^{2} v_{1}-a v_{1}}{\left(1+c y^{*}\right)\left[1+c\left(v_{1}+y^{*}\right)\right]} \\
= & \beta u_{1} v_{1}+\beta u_{1} y^{*}+\frac{\left(\beta x^{*}-\eta\right)\left(1+c y^{*}\right) c v_{1}^{2}+\left(\beta x^{*}-\eta\right)\left(1+c y^{*}\right)^{2} v_{1}-a v_{1}}{\left(1+c y^{*}\right)^{2}} \\
& -\frac{\left(\beta x^{*}-\eta\right)\left(1+c y^{*}\right) \alpha c v_{1}^{3}+\left(\beta x^{*}-\eta\right)\left(1+c y^{*}\right)^{2} \alpha v_{1}^{2}-a \alpha v_{1}^{2}}{\left(1+c y^{*}\right)^{2}+R_{2}\left(u_{1}, v_{1}\right)} \\
= & \beta u_{1} v_{1}+\beta u_{1} y^{*}+\frac{a \alpha v_{1}^{2}}{\left(1+c y^{*}\right)^{2}}+\frac{\left(\beta x^{*}-\eta\right)\left(1+c y^{*}\right)^{2} v_{1}-a v_{1}}{\left(1+c y^{*}\right)^{2}}+O_{3}\left(u_{1}, v_{1}\right)
\end{aligned}
$$

where

$$
\begin{aligned}
\alpha & =\frac{c}{1+c y^{*}} \\
R_{2}\left(u_{1}, v_{1}\right) & =\frac{\left(\beta x^{*}-\eta\right)\left(1+c y^{*}\right) c v_{1}^{2}+\left(\beta x^{*}-\eta\right)\left(1+c y^{*}\right)^{2} v_{1}-a v_{1}}{\left(1+c y^{*}\right)^{2}} \sum_{n=2}^{\infty} \frac{(-1)^{n}}{n !}(\alpha u)^{n}
\end{aligned}
$$

and

$$
O_{3}\left(u_{1}, v_{1}\right)=R_{2}\left(u_{1}, v_{1}\right) \frac{-\left(\beta x^{*}-\eta\right) \alpha c v_{1}^{3}}{1+c y^{*}}
$$


Let $u_{2}=u_{1}$ and $v_{2}=\left(\beta x^{*}-\eta-\frac{a}{\left(1+c y^{*}\right)^{2}}\right) u_{1}+\left(\beta x^{*}+q b\right) v_{1}$, then we have

$$
\begin{aligned}
& \dot{u}_{2}=-\beta u_{2}\left[\frac{v_{2}-\left(\beta x^{*}-\eta-\frac{a}{\left(1+c y^{*}\right)^{2}}\right) u_{2}}{\beta x^{*}+q b}\right]-\left(\beta y^{*}+b\right) u_{2} \\
& -\left(\beta x^{*}+q b\right)\left[\frac{v_{2}-\left(\beta x^{*}-\eta-\frac{a}{\left(1+c y^{*}\right)^{2}}\right) u_{2}}{\beta x^{*}+q b}\right] \\
& =-\frac{\beta u_{2} v_{2}}{\beta x^{*}+q b}+\frac{\beta a c y^{*}}{\left(1+c y^{*}\right)^{2}\left(\beta x^{*}+a b\right)} u_{2}^{2}-\left(\beta y^{*}+b\right) u_{2}-v_{2} \\
& +\left[\beta x^{*}-\eta-\frac{a}{\left(1+c y^{*}\right)^{2}}\right] u_{2} \\
& =-\frac{\beta u_{2} v_{2}}{\beta x^{*}+q b}+\frac{\beta a c y^{*}}{\left(1+c y^{*}\right)^{2}\left(\beta x^{*}+q b\right)} u_{2}^{2}+\operatorname{tr}\left(A\left(x^{*}, y^{*}\right)\right) u_{2}-v_{2} \\
& \dot{v}_{2}=\frac{\left[\left(\beta x^{*}-\eta\right)\left(1+c y^{*}\right)^{2}-a\right]}{\left(1+c y^{*}\right)^{2}} \dot{u}_{1}+\left(\beta x^{*}+q b\right) \dot{v}_{1} \\
& =\frac{\left[\left(\beta x^{*}-\eta\right)\left(1+c y^{*}\right)^{2}-a\right]\left[-\beta u_{1} v_{1}-\left(\beta y^{*}+b\right) u_{1}-\left(\beta x^{*}+q b\right) v_{1}\right]}{\left(1+c y^{*}\right)^{2}} \\
& +\left(\beta x^{*}+q b\right)\left[\beta u_{1} v_{1}+\beta y^{*} u_{1}+\frac{a \alpha v_{1}^{2}}{\left(1+c y^{*}\right)^{2}}+\frac{\left(\beta x^{*}-\eta\right)\left(1+c y^{*}\right)^{2} v_{1}-a v_{1}}{\left(1+c y^{*}\right)^{2}}+O_{3}\left(u_{1}, v_{1}\right)\right] \\
& =\frac{a \alpha\left(\beta x^{*}+q b\right)}{\left(1+c y^{*}\right)^{2}} v_{1}^{2}+\frac{\left.\beta(b+r)\left(1+c y^{*}\right)^{2}+a\right)}{\left(1+c y^{*}\right)^{2}} u_{1} v_{1} \\
& -\frac{\left(\beta y^{*}+b\right)\left[\left(\beta x^{*}-\eta\right)\left(1+c y^{*}\right)^{2}-a\right] u_{1}}{\left(1+c y^{*}\right)^{2}}+\left(\beta x^{*}+q b\right) \beta y^{*} u_{1}+\left(\beta x^{*}+q b\right) O_{3}\left(u_{1}, v_{1}\right) \\
& =\frac{a \alpha\left(\beta x^{*}+q b\right)}{\left(1+c y^{*}\right)^{2}} v_{1}^{2}+\frac{\left.\beta(b+r)\left(1+c y^{*}\right)^{2}+a\right)}{\left(1+c y^{*}\right)^{2}} u_{1} v_{1}+\left(\beta x^{*}+q b\right) O_{3}\left(u_{1}, v_{1}\right) \\
& =\frac{a \alpha\left(\beta x^{*}+q b\right)}{\left(1+c y^{*}\right)^{2}}\left[\frac{v_{2}(1+c y)^{2}-\left[\left(\beta x^{*}-\eta\right)\left(1+c y^{*}\right)^{2}-a\right] u_{2}}{\left(1+c y^{*}\right)^{2}(\beta x+q b)}\right]^{2} \\
& +\frac{\beta(b+r)\left(1+c y^{*}\right)^{2}+a}{\left(1+c y^{*}\right)^{2}} u_{2}\left[\frac{v_{2}(1+c y)^{2}-\left[\left(\beta x^{*}-\eta\right)\left(1+c y^{*}\right)^{2}-a\right] u_{2}}{\left(1+c y^{*}\right)^{2}(\beta x+q b)}\right]
\end{aligned}
$$




$$
\begin{aligned}
& +\left(\beta x^{*}+q b\right) O_{3}\left(u_{2}, v_{2}\right) \\
& =\zeta_{1} v_{2}^{2}+\zeta_{2} u_{2} v_{2}+\zeta_{3} u_{2}^{2}+\left(\beta x^{*}+q b\right) O_{3}\left(u_{2}, v_{2}\right)
\end{aligned}
$$

where

$$
\begin{aligned}
\zeta_{1} & =\frac{\alpha a}{\left(1+c y^{*}\right)^{2}(\beta x+q b)} \\
\zeta_{2} & =\frac{\left[\beta(b+r)\left(1+c y^{*}\right)^{2}+a\right]}{\left(1+c y^{*}\right)^{2}\left(\beta x^{*}+q b\right)}-\frac{2 \alpha a\left[\left(\beta x^{*}-\eta\right)\left(1+c y^{*}\right)^{2}-a\right]}{\left(1+c y^{*}\right)^{2}} \\
\zeta_{3} & =-\frac{\left[\beta(b+r)\left(1+c y^{*}\right)^{2}+a\right]\left[\left(\beta x^{*}-\eta\right)\left(1+c y^{*}\right)^{2}+a\right]}{\left(1+c y^{*}\right)^{4}\left(\beta x^{*}+q b\right)}
\end{aligned}
$$

By lemma (4.0.3) we have that for $a \in\left[\beta-\eta, a_{0}\right), \operatorname{tr}\left(A\left(x^{*}, y^{*}\right)\right) \neq 0$.

Let $u_{3}=\operatorname{tr}\left(A\left(x^{*}, y^{*}\right)\right) u_{2}-v_{2}$ and $v_{3}=v_{2}$, then the above system becomes

$$
\begin{aligned}
\dot{u}_{3} & =\operatorname{tr}\left(A\left(x^{*}, y^{*}\right)\right) \dot{u}_{2}-\dot{v}_{2} \\
& =-\frac{\left.\beta \operatorname{tr}\left(A\left(x^{*}, y^{*}\right)\right)\right)}{\beta x^{*}+q b} u_{2} v_{2}+\frac{\beta a c y^{*} \operatorname{tr}\left(A\left(x^{*}, y^{*}\right)\right)}{\left(1+c y^{*}\right)^{2}\left(\beta x^{*}+q b\right)} u_{2}^{2}+\operatorname{tr}\left(A\left(x^{*}, y^{*}\right)\right)^{2} u_{2} \\
& -\operatorname{tr}\left(A\left(x^{*}, y^{*}\right)\right) v_{2}-\zeta_{1} v_{2}^{2}-\zeta_{2} u_{2} v_{2}-\zeta_{3} u_{2}^{2}-\left(\beta x^{*}+q b\right) O_{3}\left(u_{2}, v_{2}\right) \\
& =-\left[\frac{\beta \operatorname{tr}\left(A\left(x^{*}, y^{*}\right)\right)}{\beta x^{*}+q b}+\zeta_{2}\right] u_{2} v_{2}+\left[\frac{\beta a c y^{*} \operatorname{tr}\left(A\left(x^{*}, y^{*}\right)\right)}{\left(1+c y^{*}\right)^{2}\left(\beta x^{*}+q b\right)}-\zeta_{3}\right] u_{2}^{2} \\
& +\operatorname{tr}\left(A\left(x^{*}, y^{*}\right)\right)^{2} u_{2}-\operatorname{tr}\left(A\left(x^{*}, y^{*}\right)\right) v_{2}-\zeta_{1} v_{2}^{2}-\left(\beta x^{*}+q b\right) O_{3}\left(u_{2}, v_{2}\right) \\
& =-\left(\frac{\beta \operatorname{tr}\left(A\left(x^{*}, y^{*}\right)\right)+\zeta_{2}\left(\beta x^{*}+q b\right)}{\left(\beta x^{*}+q b\right)} v_{3}\left[\frac{u_{3}+v_{3}}{\operatorname{tr}\left(A\left(x^{*}, y^{*}\right)\right)}\right]\right)+u_{3}+\zeta_{1} v_{3}^{2} \\
& -\left(\beta x^{*}+q b\right) O_{3}\left(u_{3}, v_{3}\right)+\left[\frac{\beta a c y^{*} \operatorname{tr}\left(A\left(x^{*}, y^{*}\right)\right)}{\left(1+c y^{*}\right)^{2}\left(\beta x^{*}+q b\right)}-\zeta_{3}\right]\left[\frac{u_{3}+v_{3}}{\operatorname{tr}\left(A\left(x^{*}, y^{*}\right)\right)}\right]^{2}
\end{aligned}
$$


Let $u=v_{3}$ and $v=u_{3}$, then the above becomes

$$
\begin{aligned}
\dot{u} & =\frac{\operatorname{tr}\left(A\left(x^{*}, y^{*}\right)\right)^{2} \zeta_{1}+\operatorname{tr}\left(A\left(x^{*}, y^{*}\right)\right) \zeta_{2}+\zeta_{3}}{\operatorname{tr}\left(A\left(x^{*}, y^{*}\right)\right)^{2}} u^{2}+\frac{\operatorname{tr}\left(A\left(x^{*}, y^{*}\right)\right) \zeta_{2}+2 \zeta_{3}}{\operatorname{tr}\left(A\left(x^{*}, y^{*}\right)\right)^{2}} u v \\
& +\frac{\zeta_{3}}{\operatorname{tr}\left(A\left(x^{*}, y^{*}\right)\right)^{2}} v^{2}+\left(\beta x^{*}+q b\right) O_{3}(v, u) \\
\dot{v} & =v-\left[\frac{\beta \operatorname{tr}\left(A\left(x^{*}, y^{*}\right)\right)+\zeta_{2}\left(\beta x^{*}+q b\right)}{\left(\beta x^{*}+q b\right)} u\left[\frac{v+u}{\operatorname{tr}\left(A\left(x^{*}, y^{*}\right)\right)}\right]+\zeta_{1} u^{2}-\left(\beta x^{*}+q b\right) O_{3}(v, u)\right. \\
& +\left[\frac{\beta a c y^{*} \operatorname{tr}\left(A\left(x^{*}, y^{*}\right)\right)}{\left(1+c y^{*}\right)^{2}\left(\beta x^{*}+q b\right)}-\zeta_{3}\right]\left[\frac{v+u}{\operatorname{tr}\left(A\left(x^{*}, y^{*}\right)\right)}\right]^{2}
\end{aligned}
$$

Where, $\varrho:=-1 \neq 0$, the result follows from lemma (2.1.2).

Theorem 4.0.5. Assume $b, r>0, c>c_{2}, q \in[0,1], \eta<\beta$ and $\beta-\eta \leq a$ then the equilibrium point $\left(x_{1}, y_{1}\right)$ is a saddle point of system (3.3.3)

Proof. From the result of Lemma (4.0.2) (1), we have

$$
\left|\mathrm{A}\left(x_{1}, y_{1}\right)\right|=\frac{y_{1}}{\left(1+c y_{1}\right)^{2}}\left[\beta(b+r) y_{1}^{2} c^{2}+2\left[\beta(b+r) y_{1}-a b\right] c+\beta(a+b+r)\right]
$$

Since $c>c_{2}$ then $\left(x_{1}, y_{1}\right)$ is a positive equilibria of the system (3.3.3) by Theorem (3.5.6).

Substitute $y_{1}$ from (3.5.10) into $\left|\mathrm{A}\left(x_{1}, y_{1}\right)\right|$ then we have

$$
\left|\mathrm{A}\left(x_{1}, y_{1}\right)\right|=\frac{y_{1}}{\left(1+c y_{1}\right)^{2}}\left[\beta(b+r) y_{1}^{2} c^{2}+\left[2 \beta(b+r) y_{1}-a b\right] c+\beta(a+b+r)\right]
$$

Let

$$
\theta\left(a, b, c, r, \beta, y_{1}\right)=\beta(b+r) y_{1}^{2} c^{2}+\left[2 \beta(b+r) y_{1}-a b\right] c+\beta(a+b+r)
$$

Consider the expression inside the square bracket together with value of $y_{1}$ from (3.5.10). 


$$
\begin{aligned}
\theta & =\beta(b+r)\left[\frac{b(\beta-\eta) c-\beta(a+b+r)-\sqrt{\Delta(c)}}{2 \beta c(b+r)}\right]^{2} c^{2} \\
& +\beta(a+b+r)+\left(2 \beta(b+r)\left[\frac{b(\beta-\eta) c-\beta(a+b+r)-\sqrt{\Delta(c)}}{2 \beta c(b+r)}\right]-a b\right) c \\
& =\frac{[b(\beta-\eta) c-\beta(a+b+r)-\sqrt{\Delta(c)}]^{2}}{4 \beta(b+r)}+\beta(a+b+r)-a b c \\
& +[b(\beta-\eta) c-\beta(a+b+r)-\sqrt{\Delta(c)}] \\
& =\frac{[b(\beta-\eta) c-\beta(a+b+r)-\sqrt{\Delta(c)}]^{2}}{4 \beta(b+r)}+b(\beta-\eta) c-a b c-\sqrt{\Delta(c)}
\end{aligned}
$$

Since

$$
\begin{aligned}
& {[b(\beta-\eta) c-\beta(a+b+r)-\sqrt{\Delta(c)}]^{2}} \\
& =([b(\beta-\eta) c-\beta(a+b+r)]-\sqrt{\Delta(c)})^{2} \\
& =[b(\beta-\eta) c-\beta(a+b+r)]^{2}-2[b(\beta-\eta) c-\beta(a+b+r)] \sqrt{\Delta(c)}+\Delta(c) \\
& =\Delta(c)+4 \beta b c(b+r)(a-\beta+\eta)-2[b(\beta-\eta) c-\beta(a+b+r)] \sqrt{\Delta(c)}+\Delta(c) \\
& =2 \Delta(c)+4 \beta b c(b+r)(a-\beta+\eta)-2[b(\beta-\eta) c-\beta(a+b+r)] \sqrt{\Delta(c)}
\end{aligned}
$$

where $\Delta(c)$ was defined from (3.5.6), so that

$$
\begin{aligned}
\theta & =\beta(a+b+r)-a b c+[b(\beta-\eta) c-\beta(a+b+r)-\sqrt{\Delta(c)}] \\
& +\frac{2 \Delta(c)+4 \beta b c(b+r)(a-\beta+\eta)-2[b(\beta-\eta) c-\beta(a+b+r)] \sqrt{\Delta(c)}}{4 \beta(b+r)} \\
& =\frac{2 \sqrt{\Delta(c)}[\sqrt{\Delta(c)}-[b(\beta-\eta) c-\beta(a+b+r)]]}{4 \beta(b+r)} \\
& +b(\beta-\eta) c-\sqrt{\Delta(c)}-a b c+b c(a-\beta+\eta)
\end{aligned}
$$




$$
\begin{aligned}
& =\frac{\sqrt{\Delta(c)}[\sqrt{\Delta(c)}-[b(\beta-\eta) c-\beta(a+b+r)]]}{2 \beta(b+r)}-\sqrt{\Delta(c)} \\
& =\sqrt{\Delta(c)}\left[\frac{\sqrt{\Delta(c)}-[b(\beta-\eta) c-\beta(a+b+r)]}{2 \beta(b+r)}-1\right] \\
& =\sqrt{\Delta(c)}\left[-\frac{[b(\beta-\eta) c-\beta(a+b+r)-\sqrt{\Delta(c)}]}{2 \beta(b+r)}-1\right] \\
& =-\sqrt{\Delta(c)}\left[\frac{[b(\beta-\eta) c-\beta(a+b+r)-\sqrt{\Delta(c)}]}{2 \beta(b+r)}+1\right]
\end{aligned}
$$

By (3.5.10) imply

$$
c y_{1}=\frac{b(\beta-\eta) c-\beta(a+b+r)-\sqrt{\Delta(c)}}{2 \beta(b+r)}
$$

so

$$
\left|\mathrm{A}\left(x_{1}, y_{1}\right)\right|=\frac{\theta y_{1}}{\left(1+c y_{1}\right)^{2}}<0
$$

since $\theta<0$ and $y_{1}>0$. The result follows from Lemma (2.1.1) (1). 


\section{Chapter 5}

\section{Conclusion}

In this thesis the SIR epidemic model with saturated treatment function is investigated with the restriction of all positive parameters involved. We find the number of equilibria for the model and there always exist a disease-free equilibrium. If $\eta<\beta, a \geq \beta-\eta$ and $c>c_{2}$ then we have two positive equilibria $\left(x_{1}, y_{1}\right)$ and $\left(x_{2}, y_{2}\right)$ or if $c=c_{2}$ then we have only one positive equilibria $\left(x^{*}, y^{*}\right)$. We showed that under suitable conditions the disease free equilibrium can be a saddle, stable node or saddle node.

For $\left(x^{*}, y^{*}\right)$ we also provided sufficient conditions on the parameters such that $\left(x^{*}, y^{*}\right)$ is a saddle node and we also proved $\left(x_{1}, y_{1}\right)$ is a saddle of the system (3.3.3).

Since we cannot determine whether $\left(x_{2}, y_{2}\right)$ is a node, stable node or focus so that we are unable to analyze the global stability of $\left(x_{2}, y_{2}\right)$. It might require a higher level of knowledge like using Dulac criterion and Poincare- Bendixon theorem and Lyapunov function which is also the goal of my future study. 


\section{Bibliography}

[1] A. A. Andronov, E. A. Leontovich, I. I. Gordon and A. G. Maier, Qualitative Theory of Second-Order Dynamical Systems, John Wiley and Sons, New York, 1973.

[2] C.R. Zhu and K.Q.Lan Phase portraits, Hopf Bifurcations and limit cycles of the Holling-Tanner models for predator-prey interactions, Nonlinear Analysis: Real World Applications, 12 (2011), 1961-1973.

[3] C. Neuhauser, System of Differential Equations, Calculus for Biology and Medicine 3rd, 11 (2011), 587-646.

[4] C.R. Zhu and K. Q. Lan, Phase portraits, Hopf bifurcations and limit cycles of Leslie-Gower predator-prey systems with harvesting rates, Discrete Contin. Dyn. Syst. Ser. B, 14 (2010), 289-306.

[5] C.R. Zhu and K.Q.Lan, Phase portraits of predator - prey systems with harvesting rates, Discrete and Continuous Dynamical Systems, 32(3) (2012), 901-933. 
[6] Linda J.S. Allen, Nonlinear Ordinary Differential Equations: Theory and Examples, An Introduction To Mathematical Biology, 5 (2007), 176232.

[7] D.Gonze and M.kaufman, Theory of non-linear dynamical systems, homepages.ulb.ac.be/ dgonze/TEACHING/nonlinear.pdf, Accessed: 08/06/2012 (2011)

[8] X. Meng and L. Chen, The dynamics of a new SIR epidemic model concerning pulse vaccination strategy, Appl. Math. Comput., 197(2008), 582-597.

[9] H. W. Hethcote, Three basic epidemiological models, Applied mathematical ecology (Trieste, 1986), 119-144, Biomathematics, 18, Springer, Berlin, 1989.

[10] X. Meng and L. Chen, The dynamics of a new SIR epidemic model concerning pulse vaccination strategy, Appl. Math. Comput., 197(2008), $582-597$.

[11] G. P. Luo, C. R. Zhu and K. Q. Lan, Dynamics of SIR epidemic models with horizontal and vertical transmissions and constant treatment rates, submitted. J. Math. Anal. Appl.

[12] W. Wang and S. Ruan, Bifurcations in an epidemic model with constant removal rate of the infectives, J. Math. Anal. Appl., 291(2004), 775-793. 
[13] Z. Hu, W. Ma and S. Ruan, Bifurcations in an epidemic model with constant removal rate of the infectives, J. Math. Anal. Appl., 291(2004), 775-793.

[14] G. Teschl, Ordinary Differential Equations and Dynamical Systems, American Mathematical Society, 2012

[15] G. P. Luo, K. Q. Lan and C. R. Zhu, Bifurcations of ratio-dependent predator-prey Holling type III systems with harvesting rates, J. Nonlinear Funct. Anal. 2014 (2014), Article ID 16.

[16] C. M. Heggerud and K. Q. Lan, Local stability analysis of ratiodependent predator-prey models with predator harvesting rates, Appl. Math. Comput., 270(2015), 349-357.

[17] S. Jana, S. K. Nandi and T. K. Kar, Complex dynamics of an SIR epidemic model with saturated incidence rate and treatment, Acta Biotheor. 64 (2016), 65-84.

[18] H. Laarabi, A. Abta and H. Hattaf, Optimal control of a delayed SIRS epidemic model with vaccination and treatment, Acta Biotheor. 63 (2015), 87-97.

[19] K. Q. Lan and C. R. Zhu, Phase portraits, Hopf bifurcations and limit cycles of the Holling-Tanner models for predator-prey interactions, Nonlinear Analysis RWA, 12(2011), 1961-1973. 
[20] L. Perko, Differential Equations and Dynamical Systems, SpringerVerlag, New York, 1996.

[21] E. Rivero-Esquivel, E. Avila-Vales, and G. Garca-Almeida, Stability and bifurcation analysis of a SIR model with saturated incidence rate and saturated treatment, Math. Comput. Simulation. 121 (2016), 109-132.

[22] T. Doan Doan, Changrong Zhu. Kunguan Lan, Phase plan analysis of the susceptible-infected, removed-susceptible (SIRS) Eidemic model with nonlinear incidence rates, J. Nonlinear Funct. Anal. 2016 (2016), Article ID 30.

[23] G. Teschl, Ordinary Differential Equations and Dynamical Systems, American Mathematical Society, 2012.

[24] T. Doan Doan, Phase portrait of an epidemic model with incident rates, 2005

[25] X. Zhang and X. Liu, Backward bifurcation of an epidemic model with saturated treatment function, J. Math. Anal. Appl. 348 (2008), 433-443.

[26] Allen, L. J. S. (2007). An introduction to mathematical biology. Upper Saddle River, NJ: Pearson/Prentice Hall, 